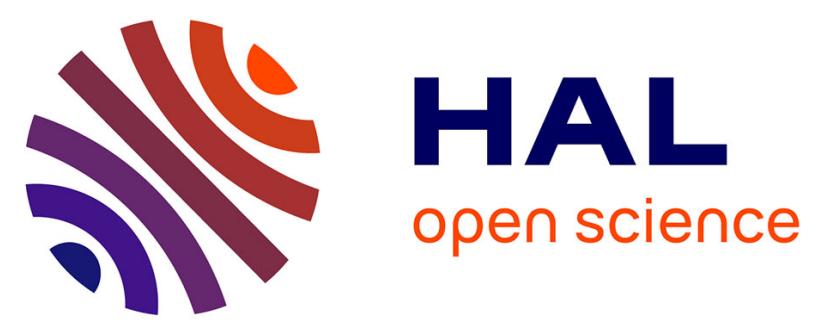

\title{
Robustness to in-domain viscous damping of a collocated boundary adaptive feedback law for an anti-damped boundary wave PDE
}

Christophe Roman, Delphine Bresch-Pietri, Christophe Prieur, Olivier Sename

\section{- To cite this version:}

Christophe Roman, Delphine Bresch-Pietri, Christophe Prieur, Olivier Sename. Robustness to in-domain viscous damping of a collocated boundary adaptive feedback law for an anti-damped boundary wave PDE. IEEE Transactions on Automatic Control, 2019, 64 (8), pp.3284-3299. 10.1109/TAC.2019.2899048 . hal-01637663v2

\section{HAL Id: hal-01637663 \\ https://hal.science/hal-01637663v2}

Submitted on 15 Jan 2019

HAL is a multi-disciplinary open access archive for the deposit and dissemination of scientific research documents, whether they are published or not. The documents may come from teaching and research institutions in France or abroad, or from public or private research centers.
L'archive ouverte pluridisciplinaire HAL, est destinée au dépôt et à la diffusion de documents scientifiques de niveau recherche, publiés ou non, émanant des établissements d'enseignement et de recherche français ou étrangers, des laboratoires publics ou privés.

\section{(ㄷ)(i) $\Theta$}

Distributed under a Creative Commons Attribution - NoDerivatives| 4.0 International 


\title{
Robustness to in-domain viscous damping of a collocated boundary adaptive feedback law for an anti-damped boundary wave PDE
}

\author{
Christophe Roman ${ }^{1}$, Delphine Bresch-Pietri ${ }^{2}$, Christophe Prieur $^{1}$ and Olivier Sename ${ }^{1}$
}

\begin{abstract}
In this paper, the robustness to model mismatch of a pre-existing collocated boundary adaptive feedback law is investigated. This control law was originally designed for an anti-damped pure wave Partial Differential Equation (PDE). Actuation and measurements are located at the same boundary. Adaptive terms account for uncertain parameters located at the anti-damped boundary, opposite to the collocated actuation and measurement. By extending and transforming the system state using, in particular, backstepping, this paper establishes that this controller is robust to sufficiently small in-domain damping. In particular both stability and attractivity (convergence) are established similarly as in the nominal case. Note moreover that, assuming that some parameters are known, the exponential stability to an attractor holds. Simulations are performed to illustrate the interest of this study to attenuate mechanical vibrations in an oil-drilling context.
\end{abstract}

\section{INTRODUCTION}

$\mathbf{K}$ EYSTONES of Partial Differential Equations include transport phenomena, the heat equation, and the wave propagation. This paper considers a special form of the latter.

The wave equation has been studied in several fields, mostly in physics regarding Maxwell equations and light propagation. Furthermore, premature failures of mechanical systems are often due to vibrations. These can be explained by the very deep structure of matter, i.e., the string-mass atomic interactions, and be modeled by a wave equation.

In this paper, we are interested in studying the robustness of a previously designed control law to a class of model mismatch. This question is of crucial interest from an application point of view. Indeed, in practice, such uncertainties are ubiquitous and robust control is thus of prime importance. However, obtaining a controller satisfying a priori robustness margins can turn out to be a very difficult problem. To handle such an objective, we propose to extend the use of a given adaptive collocated feedback law.

\section{A. Wave PDE under consideration}

We consider the following PDE system

$$
\begin{aligned}
& u_{t t}(x, t)=u_{x x}(x, t)-2 \lambda u_{t}(x, t) \\
& u_{x}(1, t)=U(t) \\
& u_{t t}(0, t)=a q u_{t}(0, t)+a\left[u_{x}(0, t)-d\right]
\end{aligned}
$$

in which $\lambda$ is an in-domain damping coefficient, $q>0$ is an unknown anti-damping boundary parameter, $d>0$ is an unknown bias, $a$ is a positive constant. The distributed variable

\footnotetext{
1 Univ. Grenoble Alpes, CNRS, Grenoble-INP, GIPSA-lab, F-38000, Grenoble, France (name.surmane@gipsa-lab.fr)

${ }^{2}$ MINES ParisTech, PSL Research University CAS - Centre Automatique et Systèmes, 60 bd Saint Michel, 75006 Paris, FRANCE (delphine.breschpietri@mines-paristech.fr)
}

of the system is $u(x, t) \in \mathbb{R}$ with $x$ the spatial variable, and $t$ the time variable. The controlled input of the system is the scalar $U(t)$. For the case in which $\lambda=0$, it has been proven (in [3]) that all eigenvalues are on the complex right-half plan, i.e., all have non negative real parts.

This system main characteristics are

(i). the unknown unstable parametric dynamics in (3) opposite to the actuation (2)

(ii). the in-domain viscous damping in (1).

The goal is to stabilize the velocity $u_{t}(x, t)$, in particular $u_{t}(0, t)$, using only the measurement of the collocated velocity $u_{t}(1, t)$ (and its history) despite in-domain damping and parameters uncertainties.

\section{B. Related work}

The distinctive feature of system (1)-(3) is the dynamical boundary (3). Note that, there exist some works on the multidimensional wave equation with dynamic boundary conditions, for example [9] and [17] where they consider a 3D setting. However, the associated literature seldom focus on stabilization (feedback law design) but on well-posedness, stability and regularity as it is the case of both references. Moreover standard literature on the one-dimensional wave equation stabilization does not consider this type of dynamics boundary (3). The most usual boundary condition is of Diriclet type, as considered in [25] where the exponential stabilization problem for a wave PDE with in-domain space-dependent term has been solved. As an intermediate step, some papers consider an anti-stable boundary. In [26], a backstepping observer based feedback is presented. Then, in [13], considering that the antistable boundary parameters are unknown, an adaptive control has been proposed. However, none of the previously mentioned works can be straightforwardly extended to encompass the dynamical boundary condition (3).

Some of the works focusing on such a problem are [8], [6], [2], [16] and [22]. In [8], the dynamical boundary is reconsidered as a PDE-ODE coupling. However, the control input is located at the dynamical boundary. Here, it is considered at the opposite boundary. In [6], both boundaries are dynamical but the wave in-domain damping is not considered. In [2], a boundary control problem, inspired by a hanging cable immersed in water is tackled. However, they do not consider viscous distributed term neither in the wave equation nor in the dynamic boundary condition, which is opposite to the Dirichlet attraction. Moreover, the controller is a full-state feedback. In [16], the wave equation is considered to model a piezoelectric stack actuator. The paper establishes the flatness of a wave PDE without distributed term and dynamic boundary conditions. Moreover, using this property an output control 
law is suggested. Finally, [22] investigates a problem similar to ours and proposes a full-state backstepping controller. But it requires the parameters $a, d, q$, and $\lambda$ to be known and full-state measurement.

\section{Paper contributions}

We are here interested in the analysis of a control law requiring only the parameter $a$ to be known, and using only measurements at the controlled boundary $(x=1)$. This paper is the logical continuation of [21] which establishes the robustness to in-domain viscous damping of the design in [3]. [3] presented a boundary velocities output adaptive feedback, i.e., using the measurement of $u_{t}(1, t)$ and $u_{t}(0, t)$.

This paper brings further developments to [4] where a collocated boundary adaptive control was designed, i.e., using the measurement of $u_{t}(1, t)$ only. Our purpose is to prove that such a control law is robust with respect to small enough indomain damping.

In other words, our objective is to prove that the system (1)-(3) is stable with the feedback law proposed in [4].

With this aim of view, we propose to build an extended system. The main difficulty in carrying out this construction is that the in-domain damping presence leads to states coupling. Moreover, this coupling modifies numerous parts of the analysis presented in [4], leading to unmatched adaptive error terms.

All these difficulties are tackled with a careful choice of the extended variables. The distributed states coupling is handled by the introduction of an estimated variable, associated to the backstepping methodology. By adding an extra state, the adaptive error term error is handled, at the expense of regularity.

The paper is organized as follows. In Section II, the problem under consideration is stated and the control design is detailed. In Section III, our robustness result is presented, i.e., the main result of this paper. Section IV is devoted to its proof. Finally, the interest of this result is illustrated through simulations of the angular velocity regulation in a drilling vibrations model.

\section{Notations}

In this paper, $|\cdot|$ is the Euclidean norm and $\|u(\cdot)\|$ is the spatial $L_{2}$-norm of a functional $[0,1] \ni x \mapsto u(x, \cdot)$, which is denoted as

$$
\|u(\cdot)\|=\sqrt{\int_{0}^{1} u(x, \cdot)^{2} d x}
$$

Sometimes, when the context is clear, the abusive notation $\|u\|$, will be used to denote $\|u(\cdot)\|$. Moreover, when the context is clear, the notation $u(t)$ will be used to denote $u(., t)$.

For $(a, b) \in \mathbb{R}^{2}$ such that $a<b$, let us define the standard projector operator on the interval $[a, b]$ as a function of two scalar arguments $f$ (denoting the parameter being updated) and $g$ (denoting the nominal update law) in the following manner

$$
\operatorname{PROJ}_{[a, b]}(f, g)=g \begin{cases}0 & \text { if } f=a \text { and } g<0 \\ 0 & \text { if } f=b \text { and } g>0 \\ 1 & \text { otherwise }\end{cases}
$$

\section{Problem Statement AND CONTROL DESIGN}

In this section, we present the problem under consideration, as well as the adaptive control law we chose to study.

Let us recall that the main features of the considered wave PDE are the in-domain damping, the unknown anti-damped boundary dynamics opposite to the control. The objective is to control the system velocity $u_{t}$, using the controlled boundary measurement $u_{t}(1, t)$ only. The following assumption will be needed throughout the paper.

Assumption 1: There exist known constants $q, \bar{q}, \underline{d}$ and $\bar{d}$ such that $\underline{q}<\bar{q}, \underline{d}<\bar{d}$ and $q \in[\underline{q}, \bar{q}], d \in[\underline{d}, \bar{d}]$.

\section{A. Presentation of the control and adaptive laws}

We consider the following control law, which was developed in [4] for the system (1)-(3) assuming $\lambda=0$,

$$
\begin{array}{r}
U(t)=-u_{t}(1, t)+\widehat{d}(t)-\left(c_{0}+\widehat{q}(t)-1\right)\left(e^{2 a(\widehat{q}(t)-1)} \mu(t)\right. \\
\left.+a \int_{t-2}^{t} e^{a(\widehat{q}(t)-1)(t-\tau)}(\eta(\tau)-\widehat{d}(t)) d \tau\right)
\end{array}
$$

in which $c_{0}>0$ is a tuning constant, $\widehat{q}$ is an estimate of the unknown parameter $q, \hat{d}$ is an estimate of the unknown parameters $d$, and $\mu$ and $\eta$ are defined as

$$
\begin{aligned}
\mu(t) & =\frac{1}{2}\left[u_{t}(1, t)+u_{t}(1, t-2)-u_{x}(1, t)+u_{x}(1, t-2)\right] \\
\eta(t) & =U(t)+u_{t}(1, t)
\end{aligned}
$$

The parameter adaptation laws are

$$
\begin{aligned}
\dot{\hat{q}}(t)= & \frac{a \gamma_{q}}{1+N(t)} \operatorname{PROJ}_{[\underline{q}, \bar{q}]}\left\{\widehat{q}(t), \mu(t)\left(\mu(t)+b_{1}\left(c_{0}+\widehat{q}(t)-1\right)\right.\right. \\
& \left.\left.\times \int_{t-2}^{t} e^{\left(a(\widehat{q}(t)-1)+\frac{1}{2}\right)(\tau-t+2)} \sigma(\tau, t) d \tau\right)\right\} \\
\dot{\hat{d}}(t)= & \frac{a \gamma_{d}}{1+N(t)} \operatorname{PROJ}_{[\underline{d}, \bar{d}]}\left\{\widehat{d}(t),-\mu(t)-b_{1}\left(c_{0}+\widehat{q}(t)-1\right)\right. \\
& \left.\times \int_{t-2}^{t} e^{\left(a(\widehat{q}(t)-1)+\frac{1}{2}\right)(\tau-t+2)} \sigma(\tau, t) d \tau\right\}
\end{aligned}
$$

in which

$$
\begin{aligned}
N(t)= & \mu(t)^{2}+b_{1} \int_{t-2}^{t} e^{\frac{\tau-t}{2}+1} \sigma(\tau, t)^{2} d \tau \\
& +b_{2} \int_{t-1}^{t} e^{\tau-t+1}(2 \mu(\tau)-\eta(\tau-2)+\widehat{d}(t))^{2} d \tau \\
\sigma(\tau, t)= & \eta(\tau)-\widehat{d}(t)+\left(c_{0}+\widehat{q}(t)-1\right)\left(e^{a(\widehat{q}(t)-1)(\tau-t+2)} \mu(t)\right. \\
& \left.+a \int_{t-2}^{\tau} e^{a(\widehat{q}(t)-1)(\tau-\chi)}(\eta(\chi)-\widehat{d}(t)) d \chi\right)
\end{aligned}
$$

The tuning parameters of the control law are $c_{0}, b_{1}, b_{2}, \gamma_{q}$ and $\gamma_{d}$. In the case where the adaptive parameters are known (i.e., $\widehat{q}=q$ and $\widehat{d}=d$ ) and without in-domain damping $(\lambda=0)$, $c_{0}$ represents the closed-loop decay rate of the velocity at $u_{t}(0, t)$.

As it has been said, the adaptive control law needs only the knowledge of $u_{t}(1, t)$, its history, and the value of the 
parameters $a$. Indeed, using the controlled boundary of the system (2) one gets that $\mu$ defined in (7) can be expressed as

$$
\mu(t)=\frac{1}{2}\left[U(t)+u_{t}(1, t)-U(t-2)+u_{t}(1, t-2)\right]
$$

The remaining parameters, i.e., $c_{0}, b_{1}, b_{2}, \gamma_{q}$ and $\gamma_{d}$ are tuning parameters and thus are set by the user. As it is usual in prediction-based design, the control law needs the history of itself and the history of the output (here $u_{t}(1, t)$ ) on a two units of time window. In the application in view, this is not a problem to use $u_{t}(1, t)$ and its past values but, actually, the key issue is to avoid using $u_{t}(0, t)$. The aforementioned control law is thus well defined. Indeed, using (2), (6), (7) and (8), one gets

$$
\begin{aligned}
& U(t)\left(1-\frac{c_{0}+\widehat{q}(t)-1}{2} e^{2 a(\widehat{q}(t)-1)}\right)=-u_{t}(1, t)\left(1+\frac{c_{0}+\widehat{q}(t)-1}{2}\right. \\
& \left.\times e^{2 a(\widehat{q}(t)-1)}\right)+\widehat{d}(t)-\left(c_{0}+\widehat{q}(t)-1\right)\left(\frac { e ^ { 2 a ( \widehat { q } ( t ) - 1 ) } } { 2 } \left(u_{t}(1, t-2)\right.\right. \\
& \left.+U(t-2))+a \int_{t-2}^{t} e^{a(\widehat{q}(t)-1)(t-\tau)}\left[U(\tau)+u_{t}(1, \tau)-\widehat{d}(t)\right] d \tau\right)
\end{aligned}
$$

Now when $\frac{c_{0}+\widehat{q}(t)-1}{2} e^{2 a(\widehat{q}(t)-1)} \neq 1$, the control law satisfies a Volterra integral of the second kind and therefore is welldefined. Moreover, in the opposite case where $c_{0}+\widehat{q}(t)-1=$ $2 e^{2 a(\widehat{q}(t)-1)}>0$, the time derivative of (14) gives also a Volterra integral of the second kind and therefore is well-defined. Nevertheless, the considered solution needs to be regular enough for $u_{t t}(1, \cdot)$ to have meaning. This is the case as we detail in Section III.

\section{B. Discussion on the control law design}

First, let us enter into the details of the control law (6). Define the Riemann variables

$$
\begin{gathered}
\zeta(x, t)=u_{t}(x, t)+u_{x}(x, t)-\widehat{d}(t) \\
\omega(x, t)=u_{t}(x, t)-u_{x}(x, t)+\widehat{d}(t)
\end{gathered}
$$

along with

$$
\begin{aligned}
W(t) & =U(t)+u_{t}(1, t)-\widehat{d}(t) \\
v(t) & =u_{t}(0, t)
\end{aligned}
$$

Then, one can express the system (1)-(3) as

$$
\begin{aligned}
\dot{v}(t) & =a(q-1) v(t)+a[\zeta(0, t)-\tilde{d}(t)] \\
\zeta_{t}(x, t) & =\zeta_{x}(x, t)-\dot{\hat{d}}(t)-\lambda(\zeta+\omega) \\
\zeta(1, t) & =W(t) \\
\omega_{t}(x, t) & =-\omega_{x}(x, t)+\dot{\widehat{d}}(t)-\lambda(\zeta+\omega) \\
\omega(0, t) & =2 v(t)-\zeta(0, t)
\end{aligned}
$$

in which $\widetilde{d}(t)=d-\widehat{d}(t)$. These equations represent two coupled transport phenomena with distributed terms, coupled with the ODE (19).

Note that $q$ is an unknown constant which we do not use neither in the control law nor the parameters update law. (19)-(23) is only a reformulation of the model at stake which indeed does depend on $q$, as $q$ is a parameter of the model.

In the case where $\lambda=0$, note that the two transport phenomena (20) and (22) are not coupled anymore. Then, for any $x$, the variable $\zeta(x, t)$ can be expressed as a delayed value of the boundary (21) (applying Lemma 6 in Appendix A-1 to (20)). This enables us to consider (19) as an input-delay system, as studied in [1], [14], and [15].

Furthermore, when $\lambda=0$, using Lemma 6 in Appendix A-1, one can get from (7)

$$
\mu(t)=u_{t}(0, t-1)
$$

Note that, considering $\widehat{q}=q$ and $\widehat{d}=d$, using (6) and (17), one can prove that

$$
W(t)=-\left(c_{0}+q-1\right) u_{t}(0, t+1)
$$

Indeed, (6) is a prediction starting from $\mu(t)=u_{t}(0, t-1)$ (according to (24)) over a two units of time horizon: one for the measurement delay (24) and one for the input delay resulting from (20). Then, the closed-loop system state $v$ satisfies

$$
\dot{v}(t)=-a c_{0} v(t), \quad \text { for } t \geqslant 1
$$

Therefore, in the nominal case $\lambda=0, \widehat{q}=q$ and $\widehat{d}=d$, exponential stabilization is achieved. In the general case, applying the certainty equivalence principle, (6) follows.

The objective of this control law is to stabilize the system (1)-(3) using the controlled boundary velocity measurement $u_{t}(1, t)$ only. This is a challenging objective, since lots of control techniques, such as backstepping, require full-state measurement, which, for a PDE system, is seldom the case in practice. Thus, many approaches use an observer (see [20] when both boundary velocities are measured). To our knowledge, such an observer has not been designed yet for the framework under consideration. For this reason, we rely on the reconstruction of a delayed value of the boundary velocity (7) (which satisfies (24) only when $\lambda=0$ ). Nevertheless, any physical relevant model has dissipative terms. Indeed, in the application considered in Section $\mathrm{V}, \lambda=0$ means that the drillstring is in a vacuum field. This emphasizes the need of the present study in order to use the considered control in real applications. Moreover, the prediction-based design is related to the Smith predictor, generally speaking the Smith predictor is not known to perform effectively with respect to model mismatch. Furthermore, $\lambda \neq 0$ prevents the use of a prediction-based design. However, note that $\lambda \neq 0$ could help for other control design, e.g. passivity. Intuitively, damping should help to stabilize the system. However as the control technique used in [4] is based on a delay representation of the system which only holds without damping, the closedloop analysis with damping is complexified here as it involves coupled PDEs.

The adaptive laws (9)-(10) result from a Lyapunov-based design, i.e., from indirect adaptive control. Moreover $\dot{\hat{d}}$ and $\dot{\hat{q}}$ are bounded by definition, due to the normalization term $N$ in (9)-(10). Besides, the projector operator allows to limit the estimated variable within its boundaries (for more details on adaptive control paradigm see [10]).

Finally, note that the control law is robust with respect to a constant input disturbance, due to the structure of (10), which can be seen as an integral term. 


\section{Problem under consideration}

We wish to study the robustness of the control law (6) designed in [4] with respect to $\lambda$, i.e., in-domain damping model mismatch. We are looking for a condition on $\lambda$ such that the system (1)-(3), using the previous control (6) and adaptive laws (9)-(10) (designed for $\lambda=0$ ), is still Lyapunov stable and convergent in a sense detailed in the following section (see Theorem 1).

\section{MAin RESUlT}

In the following, the system state is denoted as

$$
\begin{aligned}
\mathscr{X}(t)= & {\left[u(t), u_{t}(t), u(0, t), u_{t}(0, t), q-\widehat{q}(t), d-\widehat{d}(t)\right]^{T} } \\
& \in H_{2}(0,1) \times H_{1}(0,1) \times \mathbb{R}^{4}
\end{aligned}
$$

Indeed, due to the dynamical boundary (3), $u(0, t)$ and $u_{t}(0, t)$ need to be considered, in addition to $\left(u, u_{t}\right)$, to guarantee the well-posedness of the system (see [8]). Furthermore, both adaptive estimation terms are included in the state due to the choice of the (adaptive) dynamical control law. Nevertheless, as the goal of the control law is the control of velocity, we introduce two vectors

$$
\begin{gathered}
\mathscr{X}_{s}(t)=\left[u_{x}(\cdot, t), u_{t}(\cdot, t), u_{t}(0, t), q-\widehat{q}(t), d-\widehat{d}(t)\right] \\
\mathscr{X}_{a}(t)=\left[u_{x}(\cdot, t), u_{t}(\cdot, t), u_{t}(0, t), d-\widehat{d}(t)\right]
\end{gathered}
$$

The first vector is the partial state for which stabilization is achieved. The second one satisfies a convergence property, as emphasized below.

Theorem 1: Consider the closed-loop system consisting of the plant (1)-(3) satisfying Assumption 1, the control law (6) and the parameter estimation laws (9)-(10). Define the functionals $\Gamma, \Xi$ and $\Upsilon$ as

$$
\begin{aligned}
\Gamma\left(\mathscr{X}_{s}(t)\right)= & u_{t}(0, t)^{2}+\left\|u_{t}(t)\right\|^{2}+\left\|u_{x}(t)-d\right\|^{2}+\left\|u_{x t}(t)\right\|^{2} \\
& +\left\|u_{x x}(t)\right\|^{2}+(q-\widehat{q}(t))^{2}+(d-\widehat{d}(t))^{2} \\
\Xi\left(\mathscr{X}_{s}(t)\right) & =\max _{s \in[0,3]} \Gamma\left(\mathscr{X}_{s}(t-s)\right) \\
\Upsilon\left(\mathscr{X}_{a}(t)\right)= & u_{t}(0, t)^{2}+\left\|u_{t}(t)\right\|^{2}+\left\|u_{x}(t)-d\right\|^{2} \\
& +(d-\widehat{d}(t))^{2}
\end{aligned}
$$

Then, for all $c_{0}>0$, there exist $\bar{b}_{2}\left(c_{0}\right)>0, \underline{b}_{1}\left(c_{0}, \bar{b}_{2}\right)>0$, $\bar{\gamma}\left(c_{0}, \underline{b}_{1}, \bar{b}_{2}\right)>0$, such that, for

$$
\begin{aligned}
& \text { - } b_{2} \in\left(0, \bar{b}_{2}\right), \\
& \text { - } b_{1} \in\left(\underline{b}_{1}, \infty\right), \\
& \text { - } \gamma_{d}, \gamma_{q} \in(0, \bar{\gamma}) \text {, }
\end{aligned}
$$

there exists $\bar{\lambda}\left(c_{0}, b_{1}, b_{2}, \bar{\gamma}, \mathscr{X}_{s}(0)\right)>0$ such that, when $\lambda \in$ $(0, \bar{\lambda})$, it follows,

$$
\Xi\left(\mathscr{X}_{s}(t)\right) \leqslant R\left(e^{\rho \Xi\left(\mathscr{X}_{s}(0)\right)}-1\right)
$$

and

$$
\lim _{t \rightarrow \infty} \Upsilon\left(\mathscr{X}_{a}(t)\right)=0
$$

for suitable $R>0$ and $\rho>0$.

The parameters $c_{0}, b_{1}, b_{2}, \gamma_{q}$ and $\gamma_{d}$ are tuning parameters for the control law and adaptive laws. The scalar $\bar{b}_{2}, \underline{b}_{1}$, and $\bar{\gamma}$ are the parameter bounds. $\bar{\lambda}$ is the upper bound of the indomain viscous coefficient $\lambda$ for which the system (1)-(3) is stable with respect to the subspace defined as the kernel of $\Xi(\cdot)$. This is what (33) means.

As usual in adaptive control [10] (e.g. see [5] for a application of it to the wave equation), this result includes two distinct properties, (i) stability in term of the functional $\Xi$ and (ii) convergence (attractivity) in term of the functional $\Upsilon$. This is due to the fact that adaptive estimation terms may be stable but not necessarily asymptotically stable, as the term $q-\widehat{q}$.

Note that $\forall c \in \mathbb{R}, u(x, t)=d x+c$ fulfills $\Upsilon(\mathscr{X}(t))=0$. Recall that the objective of the considered adaptive control law is to stabilize $u_{t}(., t)$. As the application considered in Section V is the control of torsional vibration, we do not need to control the angular position. This is a feature inherited from the control law in [4] which is usual for the considered control problem, e.g. [23], [22], and [20]. The presented method just extends the result in [4] for in-domain viscous damping mismatch, it does not change the goal of the considered adaptive control law. Note that, if one is interested on the position stabilization, one could study the robustness mismatch of the adaptive control design in [5]. It is worth noticing that $U(t)=d$ for $u(x, t)=d x+c, \forall c \in \mathbb{R}$. This is consistent with the fact that $d$ can be seen as a feedfoward bias, and the adaptive control law $\dot{\hat{d}}$ as an integral control.

It is worth noticing that, if the adaptation parameter $\widehat{q}$ is perfectly known $(\widehat{q}=q)$, then the exponential stability of the closed-loop system (1)-(3) with the control law (6) and the adaptive law (10) in terms of the functional $\Xi$ follows ( see Lemma 2). The associated attractor is the kernel of $\Xi(\cdot)$.

\section{Comment of the closed-loop system well-posedness}

Even if the well-posedness of the closed-loop system is not tackled in this paper, the idea of its proof can be stated in three steps, as follows

(i). For each solution of the original system (of state $\mathscr{X}$ defined in (27)) there is a solution of the Extended Target system (of state $\mathscr{X}_{e}$ defined in Section IV-A6). Therefore if one proves that the extended system is well-posed, then so is the original system. Note that the Extended Target system is a coupled hyperbolic PDEs plus ODEs system.

(ii). In [18], the authors study the local well-posedness of hyperbolic PDE-ODE systems. The Extended Target system (of state $\mathscr{X}_{e}$ defined in Section IV-A6) is close to the one considered in [18]. Indeed, the first order hyperbolic PDE source terms, which are depending on ODE variables, can be canceled using a change of variable (like the one in the proof in Appendix A-1 (121)).

(iii). Using the particular property of the Extended Target system (of state $\mathscr{X}_{e}$ ), i.e., diagonal, Lipschitz and marginally stable, we can use Theorem 5.2 in [18], which states the local well-posedness. Then using the finite time blowup criterion from Theorem 5.3 in [18], and the stability property from Lemma 2 in Section IV-B, one is able to conclude the global well-posedness of the Extended Target system (of state $\mathscr{X}_{e}$ defined in Section IV-A6).

\section{PRoOF OF THEOREM 1}

The method proposed in this section to prove Theorem 1 is to define an extended system (of state $\mathscr{X}_{e}$ ), whose stability 
implies the stability of the original system in the sense of (33) in Theorem 1.

The proof is organized as follows. First, in Section Section IV-A, an extension of the system referred to as the Extended Target system, is presented. Second, we define a corresponding Lyapunov functional $V\left(\mathscr{X}_{e}\right)$, the stability of which is proved in Section IV-B. Then, in Section IV-C, two lemmas detail the equivalence properties between $V\left(\mathscr{X}_{e}\right)$ ) and $\Xi\left(\mathscr{X}_{s}\right)$ introduced in (31). Finally, the convergence with respect to the functional $\Upsilon$, defined in (32), is established in Section IV-D, and the proof of Theorem 1 is concluded in Section IV-E.

\section{A. Extended Target system}

The different steps to build the Extended Target system are listed below.

- First the system (1)-(3) is reformulated using the Riemann invariants into coupled transport phenomena with an ODE (19)-(23)

- The two transport phenomena are divided into two dynamics: the Estimated system and the Auxiliary system (Section IV-A1).

- Delayed state variables are introduced to handle the fact that the control design involves an output delay from (7) and (24) (Section IV-A2).

- A backstepping transformation is performed on a subset of these delay variables and the corresponding Target system is computed in Section IV-A3.

- A new state variable is added to handle an adaptive error term in the Lyapunov analysis (Section IV-A4).

- Finally, the system is extended with the time-and spacederivatives of the Estimated and the Auxiliary systems (Section IV-A5).

The last subsection (Section IV-A6) is a summary of the Extended Target system.

The following subsection are organized this way: first we define the variables we use to extend the system, second we compute their associated dynamics, and lastly we comment about their use.

\section{1) Estimated and Auxiliary systems}

Consider the following distributed variables

$$
\begin{aligned}
\widehat{\zeta}(x, t) & =\zeta(x, t) \\
& +\lambda \int_{x}^{1}(\zeta(\chi, t+x-\chi)+\omega(\chi, t+x-\chi)) d \chi \\
\widehat{\omega}(x, t) & =\omega(x, t)+\lambda \int_{0}^{x}(\zeta(\chi, t-x+\chi)+\omega(\chi, t-x+\chi)) d \chi \\
& -\lambda \int_{0}^{1}(\zeta(\chi, t-x-\chi)+\omega(\chi, t-x-\chi)) d \chi \\
\widetilde{\zeta}(x, t) & =\zeta(x, t)-\widehat{\zeta}(x, t) \\
\widetilde{\omega}(x, t) & =\omega(x, t)-\widehat{\omega}(x, t)
\end{aligned}
$$
of

Proposition 1: The state $v$ (18) of the ODE (19) is solution

$$
\dot{v}(t)=a(q-1) v(t)+a[\widetilde{\zeta}(0, t)+\widehat{\zeta}(0, t)-\widetilde{d}(t)]
$$

The Estimated system satisfies

$$
\begin{aligned}
\widehat{\zeta}_{t}(x, t) & =\widehat{\zeta}_{x}(x, t)-\dot{\widehat{d}}(t) \\
\widehat{\zeta}(1, t) & =W(t) \\
\widehat{\omega}_{t}(x, t) & =-\widehat{\omega}_{x}(x, t)+\dot{\widehat{d}}(t) \\
\widehat{\omega}(0, t) & =2 g(t)-\widehat{\zeta}(0, t)
\end{aligned}
$$

in which $g(t)=v(t)$, and the Auxiliary system is

$$
\begin{aligned}
\widetilde{\zeta}_{t}(x, t) & =\widetilde{\zeta}_{x}(x, t)-\lambda(\widetilde{\zeta}+\widetilde{\omega}+\widehat{\zeta}+\widehat{\omega})(x, t) \\
\widetilde{\zeta}(1, t) & =0 \\
\widetilde{\omega}_{t}(x, t) & =-\widetilde{\omega}_{x}(x, t)-\lambda(\widetilde{\zeta}+\widetilde{\omega}+\widehat{\zeta}+\widehat{\omega})(x, t) \\
\widetilde{\omega}(0, t) & =-\widetilde{\zeta}(0, t)
\end{aligned}
$$

Proof : From (19) and the definitions (35)-(38) one obtains (39).

From the definitions (35)-(36), considering the Riemann invariants (20)-(23) one gets (40)-(43). (44)-(47) are obtained in a similar manner.

We choose to denote $g=v$ in (43) because $v$ can be considered as a state and it can be express by other state variables. Therefore we kept $v$ to denote the state, and $g$ denote the values of $v$ with respect to other state variables.

These definitions follow the same ideas as in [21]. The system (20)-(23) is decomposed in two parts. The first part (40)-(43) is referred to as the Estimated system and has the same decoupled dynamics as the Nominal system (when $\lambda=0$ in (20)-(23)). It can be seen as the part where the control acts on, while the second part (44)-(47) encapsulates all the remaining dynamics. This second system is referred to as the Auxiliary system.

\section{2) Delayed states}

As the control law (6) uses a two units of time window, let us introduce some variable function of delayed state variables

$$
\begin{aligned}
& \widehat{\boldsymbol{\delta}}(x, t)=\widehat{\zeta}(x, t-1)+\widehat{d}(t-1)-\widehat{d}(t) \\
& \widehat{\beta}(x, t)=\widehat{\omega}(x, t-1)-\widehat{d}(t-1)+\widehat{d}(t)+\widetilde{\omega}(1, t-x) \\
& \widetilde{\delta}(x, t)=\widetilde{\zeta}(0, t+x-1) \\
& \widehat{\alpha}(x, t)= \begin{cases}\widehat{\delta}(2 x, t), & x \in[0,1 / 2] \\
\widehat{\zeta}(2 x-1, t), & x \in[1 / 2,1]\end{cases}
\end{aligned}
$$

Proposition 2: The variables $\mu, \widehat{\beta}, \widehat{\alpha}$, and $\widetilde{\delta}$ satisfy

$$
\begin{aligned}
\dot{\mu}(t) & =a(q-1) \mu(t)+a[\widehat{\alpha}(0, t)+\widetilde{\delta}(0, t)-\widetilde{d}(t)] \\
& -\frac{a(q-1)}{2} \widetilde{\omega}(1, t)+\frac{\widetilde{\omega}_{t}(1, t)}{2} \\
\widehat{\beta}_{t}(x, t) & =-\widehat{\beta}_{x}(x, t)+\dot{\hat{d}}(t) \\
\widehat{\beta}(0, t) & =2 \mu(t)-\widehat{\alpha}(0, t) \\
2 \widehat{\alpha}_{t}(x, t) & =\widehat{\alpha}_{x}(x, t)-2 \hat{\widehat{d}(}(t) \\
\widehat{\alpha}(1, t) & =W(t) \\
\widetilde{\delta}_{t}(x, t) & =\widetilde{\delta}_{x}(x, t) \\
\widetilde{\delta}(1, t) & =\widetilde{\zeta}(0, t)
\end{aligned}
$$


Proof : From the definition (7) of $\mu$, using the definition (15)-(16), along with the definitions (37)-(38), applying Lemma 6 in Appendix A-1 for the transport equation (40) and (42) and finally using the definition (18), one gets that

$$
\mu(t)=v(t-1)+\frac{1}{2} \widetilde{\omega}(1, t)
$$

According to the ODE satisfied by $v$ (39), and using $\widetilde{\delta}$, and $\widehat{\alpha}$, i.e., (50), and (51), one gets the following ODE at time $t-1$

$$
\dot{v}(t-1)=a(q-1) v(t-1)+a[\widehat{\alpha}(0, t)+\widetilde{\delta}(0, t)-\widetilde{d}(t)]
$$

Moreover, the time derivative of (59) gives (52).

Now, from the definition of $\widehat{\beta}$ (49), using the transport equation (42) and the associated boundary condition (43) along with the expression (59), one gets (53) and (54).

Using the definition (48), the transport equation (40), and the associated boundary condition (41), one gets (55) and (56).

Taking space and time derivatives of definition (50), one obtains the transport equation (57) associated to the boundary condition (58).

In the following, we give some comments on these additional states.

First, $\mu$ (52) is considered instead of $v$ because the state the control law (6) depends on $\mu$.

Besides, the variable $\widetilde{\delta}$ accounts for the input delay $\widetilde{\zeta}(0, t-1)$ in $(60)$. Similarly the variable $\widehat{\beta}$ represents the delay of the variable $\widehat{\omega}$.

Furthermore, the state variable $\widehat{\alpha}$ represents the history of $\widehat{\zeta}$ over a two units of time window. The idea behind gathering $\widehat{\zeta}$ and $\widehat{\delta}$ into $(51)$ is to obtain a unique distributed variable to perform a backstepping transformation (see the next section). However, we still need the variable $\widehat{\zeta}$ for two reasons. First, the boundary condition (43) depends on $\widehat{\zeta}(0, t)$. Second, in the last part of the reformulation, we need to consider the derivatives of the Estimated and Auxiliary systems (40)-(47) which are depending on $\widehat{\zeta}$ so it eases the analysis.

Finally, one can observe that the last term of (52) is not expressed in the current set of variable, i.e., $\widehat{\alpha}, \widehat{\beta}, \widetilde{\delta}, \widehat{\zeta}, \widehat{\omega}, \widetilde{\zeta}, \widetilde{\omega}, \mu, \widetilde{q}$, and $\widetilde{d}$. This is why the system representation is extended considering state derivatives in Section IV-A5.

\section{3) Backstepping transformation}

Before presenting the Target system, the control law is reformulated as follows.

Claim 1: W defined in (17) can be expressed as

$$
\begin{aligned}
W(t)= & -\left(c_{0}+\widehat{q}(t)-1\right)\left(e^{2 a(\widehat{q}(t)-1)} \mu(t)\right. \\
& \left.+2 a \int_{0}^{1} e^{2 a(\widehat{q}(t)-1)(1-\chi)} \widehat{\alpha}(\chi, t) d \chi\right)
\end{aligned}
$$

Proof: From (6)-(8) and (17), with the change of variable $\chi=t+2 x-2$, one gets

$$
\begin{aligned}
W(t)= & -\left(c_{0}+\widehat{q}(t)-1\right)\left(e^{2 a(\widehat{q}(t)-1)} \mu(t)+2 a \int_{0}^{1} e^{2 a(\widehat{q}(t)-1)(1-x)}\right. \\
& \times(W(t+2 x-2)+\widehat{d}(t+2 x-2)-\widehat{d}(t)) d x)
\end{aligned}
$$

Then, applying Lemma 6 in Appendix A-1 with $y=1$ on (55) and using (56), one obtains (61).

Consider the following backstepping transformation of $\widehat{\alpha}$

$$
\begin{aligned}
\widehat{z}(x, t)= & \widehat{\alpha}(x, t)+\left(c_{0}+\widehat{q}(t)-1\right)\left(e^{2 a(\widehat{q}(t)-1) x} \mu(t)\right. \\
& \left.+2 a \int_{0}^{x} e^{2 a(\widehat{q}(t)-1)(x-\chi)} \widehat{\alpha}(\chi, t) d \chi\right)
\end{aligned}
$$

Lemma 1: The backstepping transformation (63) together with the control law (61) transform the plant (52)-(56) into the following Target system

$$
\begin{aligned}
\dot{\mu}(t) & =-a c_{0} \mu(t)+a[\widehat{z}(0, t)+\widetilde{\delta}(0, t)+\mu(t) \widetilde{q}(t)-\widetilde{d}(t)] \\
& -\frac{a}{2}(q-1) \widetilde{\omega}(1, t)+\frac{\widetilde{\omega}_{t}(1, t)}{2} \\
2 \widehat{z}_{t}(x, t) & =\widehat{z}_{x}(x, t)+\dot{\hat{q}}(t) g_{q}(x, t)+\dot{\widehat{d}}(t) g_{d}(x, t) \\
& +[\widetilde{q}(t) \mu(t)-\widetilde{d}(t)+\widetilde{\delta}(0, t) \\
& \left.+\left[-\frac{(q-1)}{2} \widetilde{\omega}(1, t)+\frac{\widetilde{\omega}_{t}(1, t)}{2 a}\right]\right] h(x, t) \\
\widehat{z}(1, t) & =0 \\
\widehat{\beta}_{t}(x, t) & =-\widehat{\beta}_{x}(x, t)+\dot{\widehat{d}}(t) \\
\widehat{\beta}(0, t) & =\left(1+c_{0}+\widehat{q}(t)\right) \mu(t)-\widehat{z}(0, t)
\end{aligned}
$$

in which

$$
\begin{aligned}
g_{d}(x, t) & =-2-4 a\left(c_{0}+\widehat{q}(t)-1\right) \int_{0}^{x} e^{2 a(\widehat{q}(t)-1)(x-\chi)} d \chi \\
h(x, t) & =2 a\left(c_{0}+\widehat{q}(t)-1\right) e^{2 a(\widehat{q}(t)-1) x} \\
g_{q}(x, t) & =2 e^{2 a(\widehat{q}(t)-1) x} \mu(t)+4 a \int_{0}^{x} e^{2 a(\widehat{q}(t)-1)(x-s)} \widehat{\alpha}(s, t) d s \\
& +\left(c_{0}+\widehat{q}(t)-1\right)\left(4 a x e^{2 a(\widehat{q}(t)-1) x} \mu(t)\right. \\
& \left.+8 a^{2} \int_{0}^{x}(x-\chi) e^{2 a(\widehat{q}(t)-1)(x-\chi)} \widehat{\alpha}(\chi, t) d \chi\right)
\end{aligned}
$$

and in which $\widehat{\alpha}$ can be expressed via the inverse backstepping transformation

$$
\begin{aligned}
\widehat{\alpha}(x, t)= & \widehat{z}(x, t)-\left(c_{0}+\widehat{q}(t)-1\right)\left(e^{-2 a c_{0} x} \mu(t)\right. \\
& \left.+2 a \int_{0}^{x} e^{-2 a c_{0}(x-\chi)} \widehat{z}(\chi, t) d \chi\right)
\end{aligned}
$$

Proof: The proof is established from the time and space derivatives of (63), using the expressions (55)-(56) and (52). As the computations are fairly standard, they are omitted.

\section{4) Adaptive error term}

Consider the following variable

$$
\widetilde{d}_{\mu}(t)=-a c_{0} \mu(t)-a \widetilde{d}(t)
$$

Proposition 3: The dynamics of $\widetilde{d}_{\mu}$ is

$$
\begin{aligned}
\dot{\tilde{d}}_{\mu}(t) & =-a c_{0} \widetilde{d}_{\mu}(t)-a^{2} c_{0}[\widehat{z}(0, t)+\widetilde{\delta}(0, t)+\mu(t) \widetilde{q}(t) \\
& \left.-\frac{1}{2}(q-1) \widetilde{\omega}(1, t)+\frac{1}{2 a} \widetilde{\omega}_{t}(1, t)\right]+a \dot{\hat{d}}(t)
\end{aligned}
$$


and $g$ in (43) can be expressed as

$$
\begin{aligned}
g(t)= & e^{a(q-1)} \mu(t)-e^{a(q-1)} \frac{\widetilde{\omega}(1, t)}{2} \\
& +a \int_{0}^{1} e^{a(q-1)(1-\chi)}[\widehat{\alpha}(\chi / 2, t)+\widetilde{\delta}(\chi, t)] d \chi \\
& +\left[c_{0} \mu(t)+\frac{\widetilde{d}_{\mu}(t)}{a}\right]\left[\frac{e^{a(q-1)}-1}{q-1}\right]
\end{aligned}
$$

in which $\widehat{\alpha}$ is expressed using the inverse backstepping transformation (72).

Proof: Computing the time derivative of (73) and using (64), one gets (74).

Using a prediction of the ODE (60), Lemma 6 in Appendix A-1 for the transport phenomena (55) and (57), and thanks to the definition (59), one obtains

$$
\begin{aligned}
g(t) & =v(t)=e^{a(q-1)} \mu(t)-\frac{e^{a(q-1)}}{2} \widetilde{\omega}(1, t)+a \int_{0}^{1} e^{a(q-1)(1-\chi)} \\
& \times[\widehat{\alpha}(\chi / 2, t)+\widetilde{\delta}(\chi, t)] d \chi-\widetilde{d}(t)\left[\frac{e^{a(q-1)}-1}{q-1}\right]
\end{aligned}
$$

Finally, using (73), one gets (75).

A first objective of this proposition is to reformulate the function $g$ in terms of the variables of interest, that is $\mu, \widehat{z}$, $\widehat{\beta}, \widehat{\zeta}, \widehat{\omega}, \widetilde{\zeta}, \widetilde{\omega}, \widetilde{\delta}, \widetilde{q}$, and $\widetilde{d}$. A second objectif is to handle the last term of (76) involving a $\widetilde{d}(t)$. Usually, the update law $\dot{\hat{d}}$ could be designed to cope with this term. However, in the nominal case $\lambda=0$ in [4], the Lyapunov analysis was carried out in a cascaded manner. First the stability of $\widehat{z}, \mu$, and $\widehat{\beta}$ is established. Secondly using Grönwall's inequality the stability of $\widehat{\omega}$ is obtained (for more details see [4]).

Here, we cannot use the same developments, due the interconnections between the variables generated by the in-domain damping. This compels us to perform a Lyapunov analysis of the entire system, and to deal with the $\widetilde{d}$ term appearing in (76). The additional variable $\widetilde{d}_{\mu}$ is thus introduced to overcome this difficulty.

\section{5) Introduction of the first-order derivatives of the Estimated and Auxilary systems}

Note that (64) and (74) are both depending on $\widetilde{\omega}_{t}(1, t)$. To get that type of term in the Lyapunov analysis we have to take under consideration the dynamic of $\widetilde{\omega}_{t}$. As presented in the sequel, the dynamic of $\widetilde{\omega}_{t}$ is coupled with the dynamic of $\widetilde{\zeta}_{t}, \widehat{\omega}_{x}$ and $\widehat{\zeta}_{x}$. Therefore we extend the system by considering derivatives of the state variables of the Estimated and Auxiliary systems (40)-(47), as follows.

Proposition 4: The dynamics of $\widehat{\zeta}_{x}$ and $\widehat{\omega}_{x}$ are

$$
\begin{aligned}
\widehat{\zeta}_{x t}(x, t) & =\widehat{\zeta}_{x x}(x, t) \\
\widehat{\zeta}_{x}(1, t) & =\dot{W}(t)+\dot{\widehat{d}}(t) \\
\widehat{\omega}_{x t}(x, t) & =-\widehat{\omega}_{x x}(x, t) \\
\widehat{\omega}_{x}(0, t) & =-2 \dot{g}(t)+\widehat{\zeta}_{x}(0, t)
\end{aligned}
$$

in which the expressions of the time derivative of $g$ and $W$ are given in Appendix B. The dynamics of $\widetilde{\zeta}_{t}$ and $\widetilde{\omega}_{t}$ are

$$
\begin{aligned}
\widetilde{\zeta}_{t t}(x, t) & =\widetilde{\zeta}_{x t}(x, t)-\lambda\left(\widetilde{\zeta}_{t}+\widetilde{\omega}_{t}+\widehat{\zeta}_{x}-\widehat{\omega}_{x}\right)(x, t) \\
\widetilde{\zeta}_{t}(1, t) & =0 \\
\widetilde{\omega}_{t t}(x, t) & =-\widetilde{\omega}_{x t}(x, t)-\lambda\left(\widetilde{\zeta}_{t}+\widetilde{\omega}_{t}+\widehat{\zeta}_{x}-\widehat{\omega}_{x}\right)(x, t) \\
\widetilde{\omega}_{t}(0, t) & =-\widetilde{\zeta}_{t}(0, t)
\end{aligned}
$$

Proof: Taking a space-derivative of the Estimated system transport (40) and (42), one gets respectively (77) and (79). From (40) evaluated for $x=1$ and the boundary condition (41), one gets (78). With a similar argument on (42) and (43), (80) follows.

Adding the Estimated system transport phenomena (40) and (42), one obtains

$$
\widehat{\zeta}_{t}+\widehat{\omega}_{t}=\widehat{\zeta}_{x}-\widehat{\omega}_{x}
$$

Thus, the time derivative of the Auxiliary system (44)-(47) gives (81)-(84).

The state variables of the Extended Target system are detailed explicitly in the next section.

\section{6) Summary of the Extended Target system}

To summarize, the Extended Target system whose state is

$$
\begin{aligned}
& \mathscr{X}_{e}(t)=[\mu(t), \widehat{z}(t), \widehat{\beta}(t), \widetilde{\delta}(t), \widehat{\zeta}(t), \widehat{\omega}(t), \widetilde{\zeta}(t), \\
& \left.\widetilde{\omega}(t), \widetilde{d}_{\mu}(t), \widehat{\zeta}_{x}(t), \widehat{\omega}_{x}(t), \widetilde{\zeta}_{t}(t), \widetilde{\omega}_{t}(t), \widetilde{q}(t), \widetilde{d}(t)\right]^{T} \\
& \quad \in\left\{\mathbb{R}, L_{2}(0,1)^{3}, H_{1}(0,1)^{4}, \mathbb{R}, L_{2}(0,1)^{4}, \mathbb{R}^{2}\right\}
\end{aligned}
$$

consists of (64)-(68), (57)-(58), (40)-(47), (77)-(84), and (74), with the update laws (9)-(10).

Figure 1 illustrates the different steps detailed previously to build the Extended Target system.

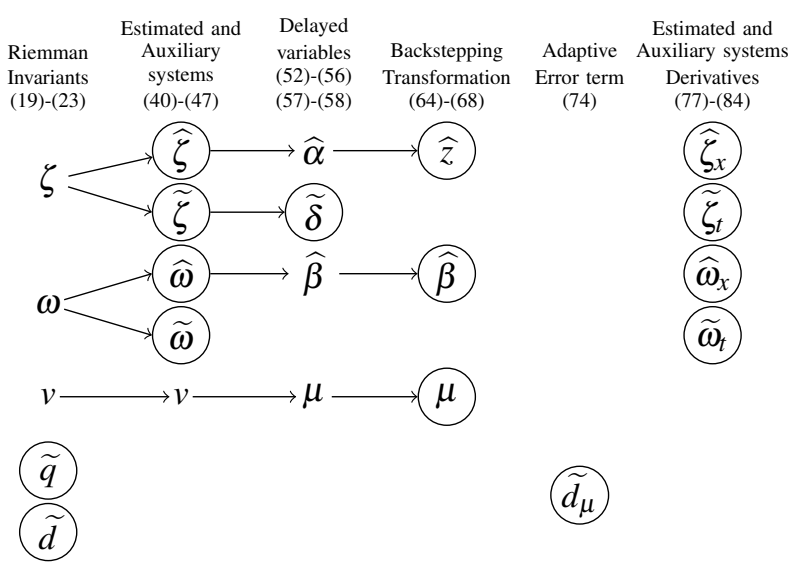

Fig. 1. Schematic view of the successive transformations and extensions to obtain the Extended Target system, variables of which are encircled.

\section{B. Lyapunov analysis}

This section focus on the stability analysis of the Extended Target system. First, we formulate the following claim 
Claim 2: The estimation laws (9)-(10) can be rewritten as

$$
\begin{aligned}
& \dot{\hat{q}}(t)=\frac{a \gamma_{q}}{1+N(t)} \operatorname{PROJ}_{[\underline{q}, \bar{q}]}\left\{\widehat{q}(t), \mu(t)\left(\mu(t)+2 b_{1}\right.\right. \\
& \left.\left.\times\left(c_{0}+\widehat{q}(t)-1\right) \int_{0}^{1} e^{2\left(a(\widehat{q}(t)-1)+\frac{1}{2}\right) x} \widehat{z}(x, t) d x\right)\right\} \\
& \dot{\hat{d}}(t)=-\frac{a \gamma_{d}}{1+N(t)} \operatorname{PROJ}_{[\underline{d}, \bar{d}]}\left\{\widehat{d}(t), \mu(t)+2 b_{1}\right. \\
& \left.\times\left(c_{0}+\widehat{q}(t)-1\right) \int_{0}^{1} e^{2\left(a(\widehat{q}(t)-1)+\frac{1}{2}\right) x} \widehat{z}(x, t) d x\right\} \\
& N(t)=\mu(t)^{2}+2 b_{1} \int_{0}^{1} e^{x} \widehat{z}(x, t)^{2} d x+b_{2} \int_{0}^{1} e^{1-x} \widehat{\beta}(x, t)^{2} d x
\end{aligned}
$$

in which $\widehat{\beta}$ is defined in (49) and $\widehat{z}$ in (63).

Proof: The proof follows arguments similar to the ones of Claim 1 in Section IV-A3.

Then, one is able to establish the following key lemma.

Lemma 2: Let us consider the Lyapunov functional

$$
V\left(\mathscr{X}_{e}(t)\right)=V_{1}(t)+V_{2}(t)+V_{3}(t)+V_{4}(t)+V_{5}(t)
$$

with

$$
\begin{aligned}
V_{1}(t) & =\log (1+N(t))+\frac{\widetilde{q}(t)^{2}}{\gamma_{q}}+\frac{\widetilde{d}(t)^{2}}{\gamma_{d}} \\
V_{2}(t) & =b_{3} \int_{0}^{1} e^{x} \widehat{\zeta}(x, t)^{2} d x+b_{4} \int_{0}^{1} e^{1-x} \widehat{\omega}(x, t)^{2} d x \\
V_{3}(t) & =b_{5} \int_{0}^{1} e^{x} \widetilde{\zeta}(x, t)^{2} d x+b_{6} \int_{0}^{1} e^{1-x} \widetilde{\omega}(x, t)^{2} d x \\
& +b_{7} \int_{0}^{1} e^{x} \widetilde{\delta}(x, t)^{2} d x \\
V_{4}(t) & =b_{8} \widetilde{d}_{\mu}(t)^{2} \\
V_{5}(t) & =b_{9} \int_{0}^{1} e^{x} \widehat{\zeta}(x, t)^{2} d x+b_{10} \int_{0}^{1} e^{1-x} \widehat{\omega}_{x}(x, t)^{2} d x \\
& +b_{11} \int_{0}^{1} e^{x} \widetilde{\zeta}_{t}(x, t)^{2} d x+b_{12} \int_{0}^{1} e^{1-x} \widetilde{\omega}_{t}(x, t)^{2} d x
\end{aligned}
$$

in which $N$ is expressed as (89), and $b_{3}, \ldots, b_{12}>0$.

For all $c_{0}>0$, there exist $\bar{b}_{2}\left(c_{0}\right)>0, \underline{b}_{1}\left(c_{0}, \bar{b}_{2}\right)>0$, $\bar{\gamma}\left(c_{0}, \underline{b}_{1}, \bar{b}_{2}\right)>0$, such that, for all

$$
\begin{aligned}
& \text { - } b_{2} \in\left(0, \bar{b}_{2}\right), \\
& \text { - } b_{1} \in\left(\underline{b}_{1}, \infty\right), \\
& \text { - } \gamma_{d}, \gamma_{q} \in(0, \bar{\gamma}) \text {, }
\end{aligned}
$$

there exist $b_{i}>0, i \in\{3, \ldots, 12\}$ and $\bar{\lambda}\left(c_{0}, b_{1}, b_{2}, \bar{\gamma}, \mathscr{X}(0)\right)>0$, such that, for all $\lambda \in(0, \bar{\lambda})$, it follows

$$
\begin{aligned}
& \dot{V}(t) \leqslant-\frac{\zeta}{1+N(t)}\left[\widehat{z}(0, t)^{2}+\widetilde{\delta}(0, t)^{2}+\mu(t)^{2}+\widetilde{d}_{\mu}(t)^{2}\right. \\
& +\widehat{\zeta}(0, t)^{2}+\widetilde{\zeta}(0, t)^{2}+\|\widehat{z}\|^{2}+\|\widehat{\beta}\|^{2}+\|\widehat{\zeta}\|^{2}+\|\widetilde{\zeta}\|^{2}+\|\widehat{\omega}\|^{2} \\
& \left.+\|\widetilde{\omega}\|^{2}+\|\widetilde{\delta}\|^{2}+\left\|\widehat{\zeta}_{x}\right\|^{2}+\left\|\widetilde{\zeta}_{x}\right\|^{2}+\left\|\widehat{\omega}_{t}\right\|^{2}+\left\|\widetilde{\omega}_{t}\right\|^{2}\right]
\end{aligned}
$$

for a suitable $\varsigma>0$, and

$$
V\left(\mathscr{X}_{e}(t)\right) \leqslant V\left(\mathscr{X}_{e}(0)\right), \quad t \geqslant 0
$$

Proof: The proof is given in Appendix C

\section{Relation between the functionals $\Gamma\left(\mathscr{X}_{s}\right)$ and $V\left(\mathscr{X}_{e}\right)$}

In order to establish the stability condition (33) of Theorem 1, we formulate the following two lemmas.

Lemma 3: Consider $\Gamma$ defined in (30) and $V$ defined in (90). There exists $R>0$, such that

$$
\Gamma\left(\mathscr{X}_{s}(t)\right) \leqslant R\left(e^{V\left(\mathscr{X}_{e}(t)\right)}-1\right)
$$

Proof: The details of the proof are given in Appendix D.

To establish Theorem 1, we also need to bound $V$ by a function of $\Gamma$. This is the purpose of the next lemma.

Lemma 4: Consider $\Gamma$ defined in (30) and $V$ defined in (90). There exists $\rho>0$ such that

$$
V\left(\mathscr{X}_{e}(t)\right) \leqslant \rho \max _{s \in[0,3]} \Gamma\left(\mathscr{X}_{s}(t-s)\right)
$$

Proof: The proof is given in Appendix D

\section{Convergence analysis}

We conclude on the convergence with respect to the functional $\Upsilon(32)$.

Lemma 5: $v(t),\|\widehat{\omega}(t)\|,\|\widehat{\zeta}(t)\|,\|\widetilde{\zeta}(t)\|$, and $\|\widetilde{\omega}(t)\|$ tend to zero as $t$ tends to infinity.

The proof, based on Barbalat's lemma, is presented in Appendix E.

\section{E. Conclusion on the proof of Theorem 1}

Gathering (98) from Lemma 3 and (99) from Lemma 4, one gets the existence of $R>0$ and $\rho>0$ such that

$$
\Gamma\left(\mathscr{X}_{s}(t)\right) \leqslant R\left(e^{\rho \max _{s \in[0,3]} \Gamma\left(\mathscr{X}_{s}(-s)\right)}-1\right),
$$

Then, (100) along with (97) from Lemma 2 give the stability result (33) in Theorem 1.

Finally, using Lemma 5, (15)-(16) and (37)-(38), it follows that $u_{t}(0, t)=v(t),\left\|u_{t}(t)\right\|$ and $\left\|u_{x}-\widehat{d}(t)\right\|$ and also $d-\widehat{d}(t)$ tend to zero as $t$ tends to infinity. In other words, (34) holds. This concludes the proof of Theorem 1 .

\section{APPLICATION TO DRILLING TORSIONAL VIBRATIONS}

For illustration purposes, the control law presented in Section II is applied to a nonlinear drilling model, presented in the next section.

\section{A. Drilling context and nonlinear model}

One of the possible applications of the control law (6) associated with the wave equation model (1)-(3) is to attenuate the torsional vibrations occurring in drilling facilities (e.g. [3], [4], and [22]). Such vibrations can lead to the so called stickslip phenomenon [12]. Indeed, the friction at the bottom of the hole, between the rock and the drillbit, forces sometimes the bit to stop, while the surface is still rotating. After some time, the bit will start moving again at velocity higher than the top velocity (see Figure 5 before $15 \mathrm{~s}$ ). This torsional dynamics can be modeled by a wave equation with a nonlinear boundary condition $u_{t t}(0, t)=a F\left(u_{t}(0, t)\right)+a u_{x}(0, t)$ (see [23], [24]), accounting for the friction between the drillbit and 


\begin{tabular}{cll} 
Symbol & Description & Value \\
\hline \hline$L$ & Length of the drillstring & $2000 \mathrm{~m}$ \\
$J$ & Drillstring second moment of area & $1.19 \mathrm{e}-5 \mathrm{~m}^{4}$ \\
$G$ & Shear modulus & $79.3 \mathrm{e} 10 \mathrm{~N} / \mathrm{m}^{2}$ \\
$I$ & Drillstring inertia's moment per length unit & $9.5 \mathrm{e}-2 \mathrm{~kg} . \mathrm{m}^{2}$ \\
$I_{b}$ & BHA moment of inertia & $311 \mathrm{~kg} . \mathrm{m}$ \\
$D_{d p}$ & Outer diameter of the drill pipe & $1.27 \mathrm{e}-1 \mathrm{~m}$ \\
$d_{d p}$ & Inner diameter of the drill pipe & $1.08 \mathrm{e}-1 \mathrm{~m}$ \\
$\mu_{m}$ & Field viscous coefficient & $\{0,10,20\} \mathrm{Pa} . \mathrm{s}$ \\
$c_{b}$ & Sliding torque coefficient & $2 \mathrm{e} 3 \mathrm{~N} . \mathrm{m.s} / \mathrm{rad}$ \\
$T_{t o b}$ & Torque-on-the-bit parameter & $7.5 \mathrm{e} 2 \mathrm{~N} . \mathrm{m}$ \\
$\alpha_{1}, \alpha_{2}, \alpha_{3}$ & Friction parameters & $5.5 ; 2.2 ; 3500$ \\
$\gamma$ & Damping parameter & $0.03 \mathrm{~N} . \mathrm{m} . \mathrm{s} / \mathrm{rad}$
\end{tabular}

These value are taken from [22] and [11]. The friction phenomenon is described by the model in [27].

TABLE I

PARAMETERS VALUES OF THE NONLINEAR MODEL USED IN SIMULATION

the rock. Even if there exist phenomenological expressions of this friction $F$ (see [27], [24] and [19]), they depend on some parameters, such as the weight on the bit, drilling mud properties, and the nature of the rock. So they may change during operation. This is the reason why using an adaptive controller is of high interest for this application.

Following [24], the nonlinear dynamical model of the drillstring rotatory angle is denoted $\theta(\xi, \mathrm{t})$ at length $\xi$ and time $t$. By convention, the top boundary is at $\xi=0$ and the bottom boundary at $\xi=L$, and the torsional dynamics can be modeled by

$$
G J \theta_{\xi \xi}(\xi, \mathrm{t})-I \theta_{\mathrm{tt}}(\xi, \mathrm{t})-\Lambda \theta_{\mathrm{t}}(\xi, \mathrm{t})=0
$$

along with the boundary conditions

$$
\begin{aligned}
& G J \theta_{\xi}(0, \mathrm{t})=c_{\alpha}\left(\theta_{\mathrm{t}}(0, \mathrm{t})-\Omega(\mathrm{t})\right) \\
& I_{b} \theta_{\mathrm{tt}}(L, \mathrm{t})=-G J \theta_{\xi}(L, \mathrm{t})-T_{B I T}\left(\theta_{\mathrm{t}}(L, \mathrm{t})\right)
\end{aligned}
$$

in which $\Omega(t)$ is the angular velocity of the rotatory table rotor the actual actuator at time $t, T_{B I T}$ is the nonlinear rock-onthe-bit friction term and other constants are listed in Table I. Note that there exist alternative models of this phenomenon, such as proposed in [23] which takes into account the axial vibrations.

Following [22], we consider the changes of variables

$$
\begin{aligned}
\xi & =L(1-x) \\
\mathrm{t} & =L \sqrt{\frac{I}{G J}} t \stackrel{\Delta}{=} c_{t} t \\
u(x, t) & =\theta(\xi, \mathrm{t}) \\
U(t) & =\frac{L c_{\alpha}}{G J}\left(\Omega\left(c_{t} t\right)-\frac{1}{c_{t}} u_{t}(1, t)\right) \\
F(\cdot) & =-\frac{L}{G J} T_{B I T}\left(\frac{\cdot}{c_{t}}\right)
\end{aligned}
$$

with the following constants

$$
a=L \frac{I}{I_{B}}, \quad 2 \lambda=\frac{\Lambda L}{\sqrt{G J I}}
$$

This allows to rewrite (101)-(103) as

$$
\begin{aligned}
& u_{t t}(x, t)=u_{x x}(x, t)-2 \lambda u_{t}(x, t) \\
& u_{x}(1, t)=U(t) \\
& u_{t t}(0, t)=a F\left(u_{t}(0, t)\right)+a u_{x}(0, t)
\end{aligned}
$$

Now, we consider a first order Taylor approximation of $F($. around an equilibrium $u_{t}^{\text {ref }}$, i.e.,

$$
F\left(u_{t}(0, t)\right)=q\left(u_{t}(0, t)-u_{t}^{\mathrm{ref}}\right)-d
$$

in which $d=-F\left(u_{t}^{\text {ref }}\right)$ and $q=\dot{F}\left(u_{t}^{\text {ref }}\right)$. Then, assuming $u_{t}^{\text {ref }}=0$ for the sake of conciseness, the torsional dynamics model can be reformulated under the form (1)-(3).

For simulation, the friction term $T_{B I T}($.$) is taken as (see$ [27])

$$
T_{B I T}(\chi)=\gamma \chi+\frac{2 T_{t o b}}{\pi}\left(\alpha_{1} \chi e^{-\alpha_{2}|\chi|}+\arctan \left(\alpha_{3} \chi\right)\right)
$$

where the parameter values are gathered in Table I.

The drill pipe is a hollow cylinder of outer diameters $D_{d p}$ and inner diameter $d_{d p}$. The drill is in contact with a viscous field, of viscous dynamical coefficient $\mu_{m}$. Direct computation gives the associated damping coefficient

$$
\Lambda=\mu_{m} \frac{\pi}{2}\left(D_{d p}^{2}+d_{d p}^{2}\right)
$$

In Figure 2, we provide a brief illustration of the simulation scheme.

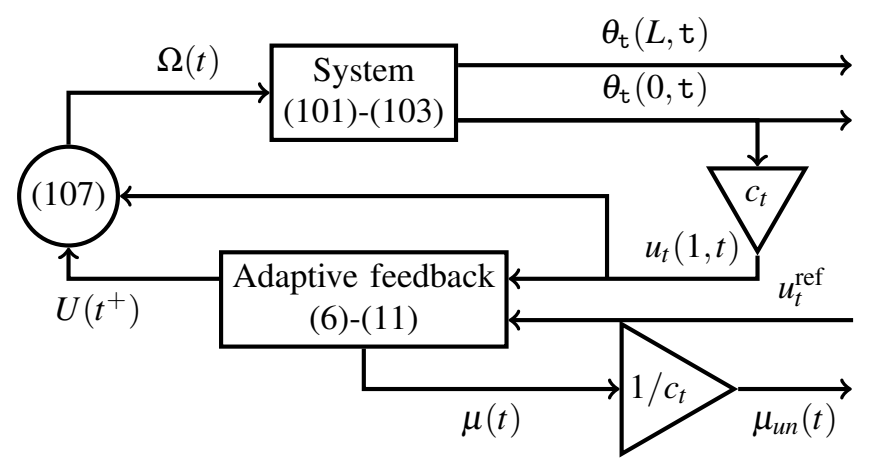

Fig. 2. Simulation scheme.

Simulation are performed in the sequel for $\lambda=0$ (nominal case), $\lambda=0.45$ and $\lambda=0.9$. It is worth noting that all simulations presented below have been performed using the nonlinear model (101)-(103).

\section{B. Velocity regulation of the nonlinear model}

Here, the control law (6) and adaptation laws (9)-(11) are not used for stabilization but for regulation, i.e., we wish to stabilize $u_{t}(x, t)-u_{t}^{\text {ref }}$ instead of $u_{t}(x, t)$. Note that in all simulation cases, the control law is turned on at $t=15 s . c_{0}$ is chosen such as $a c_{0}=1$. Besides, we chose $b_{2}=10^{-4}, b_{1}=1$, $\gamma_{d}=0.5$ and $\gamma_{q}=0.01$.

The reference $u_{t}^{\text {ref }}$ is taken such that the unnormalized desired velocity is $\theta_{t}^{\text {ref }}=5 \mathrm{rad} / \mathrm{s}$. The top and bottom velocities $\theta_{\mathrm{t}}(0, t)$ and $\theta_{\mathrm{t}}(L, t)$ along with the unnormalized equivalent of $\mu$ defined as

$$
\mu_{\mathrm{un}}(\mathrm{t})=\frac{\mu(t)}{c_{t}}
$$

are all displayed in Figures 3,5 , and 8 for $\lambda=0,0.45,0.9$ respectively. The estimations of $d$ and $q$ are displayed in Figure 7 for the case $\lambda=0.45$. 


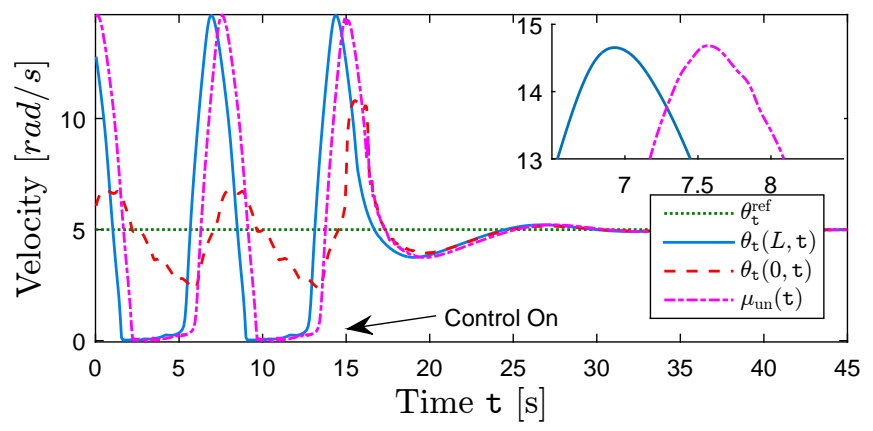

Fig. 3. Simulation of the top, bottom and delayed bottom estimated velocities for $\lambda=0$, the nominal case for which the control has been developed (see [4]).
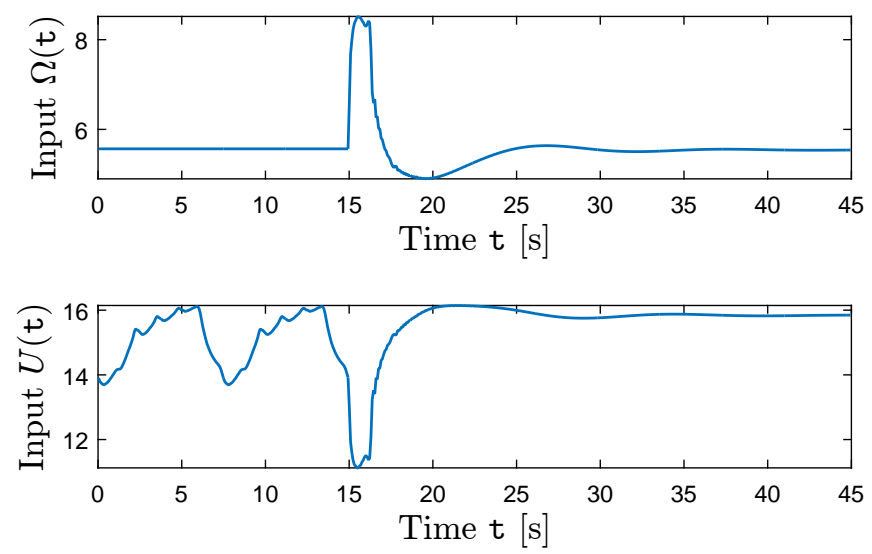

Fig. 4. Time response of the control laws $\Omega(t)$ and $U(t)$ in the case $\lambda=0$.

One can notice that the oscillations existing in the openloop phase, i.e., before $15 s$ in Figures 3 and Figure 5, are mitigated by the application of the control law and regulation is obtained.

In Figure 3, as excepted when $\lambda=0$ from (24), (106), and (116), one gets $\mu_{\mathrm{un}}(\mathrm{t})=\theta_{\mathrm{t}}\left(L, \mathrm{t}-c_{t}\right)$. Moreover, the top and bottom velocities $\theta_{\mathrm{t}}(0, \mathrm{t})$ and $\theta_{\mathrm{t}}(L, \mathrm{t})$ reach $95 \%$ of the reference, at respectively 23.54 and $23.37 s$, which are reasonable performance compared to the oscillation period. The time responses of the associated control laws $\Omega(t)$ and $U(\mathrm{t})$ are displayed in Figure 4.

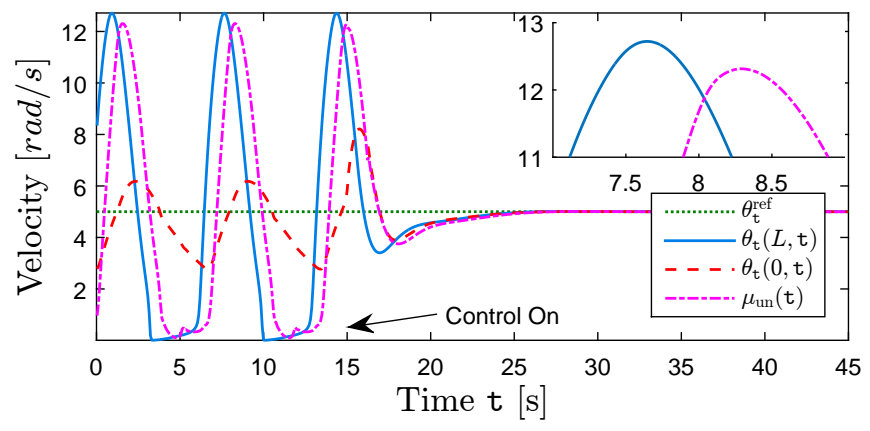

Fig. 5. Simulation of the top, and bottom and delayed bottom estimated velocities for $\lambda=0.45$.
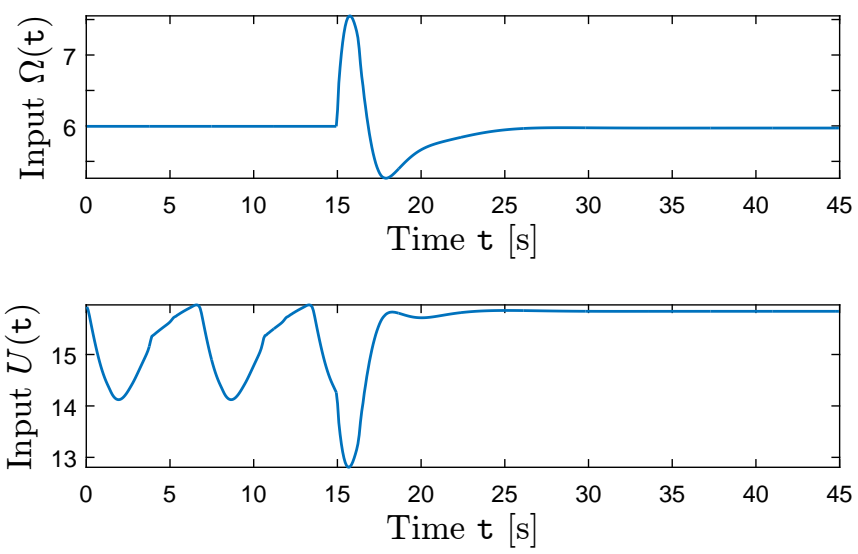

Fig. 6. Time response of the control laws $\Omega(\mathrm{t})$ and $U(\mathrm{t})$ in the case $\lambda=0.45$.

In Figure $5(\lambda=0.45)$, the top and bottom velocities $\theta_{\mathrm{t}}(0, \mathrm{t})$ and $\theta_{\mathrm{t}}(L, \mathrm{t})$ reach $95 \%$ of the reference at respectively 22.00 and $21.74 \mathrm{~s}$. One observes that these settling times are close to the nominal case. Here, as excepted from (86), (106), and (116), $\mu_{\mathrm{un}}(t)$ is only an approximation of $\theta_{\mathrm{t}}\left(L, \mathrm{t}-c_{t}\right)$. The time responses of the associated control laws $\Omega(\mathrm{t})$ and $U(\mathrm{t})$ are displayed in Figure 6.

The fact that the system for $\lambda=0.45$ in Figure 5 has a settling time lower that in the nominal case $(\lambda=0$ in Figure 3) can probably be explained by the fact that the respective velocity values are not the same when the controller is turned on. Moreover, notice that the magnitude of the oscillations before $t=15 s$ is lower in Figure 5 (case $\lambda=0.45$ ) than in Figure 3 (case $\lambda=0$ ), which is clearly explained by the fact that the in-domain damping adds dissipation.
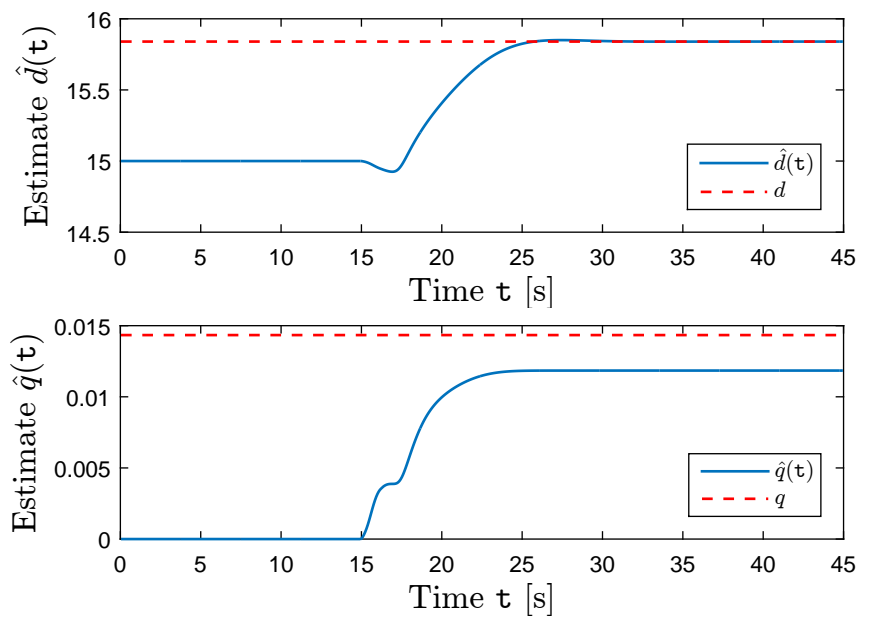

Fig. 7. Evolution of the parameter estimates $\widehat{d}$ and $\widehat{q}$ for $\lambda=0.45$.

In Figure 7, as expected, $\widehat{d}(\mathrm{t})$ converges to $d$. Even if velocity regulation is obtained, one can observe that the estimation $\widehat{q}(\mathrm{t})$ does not converge to the value of $q$. Note that this latter observation does not contradict the conclusion of Theorem 1. This is deeply linked with our control goal, i.e., regulation (stabilization). Indeed, the control laws associated with this goal are not frequentially "rich" enough (as pictured in Figure 4 and Figure 6) for $\widehat{q}(t)$ to converge towards $q$. 


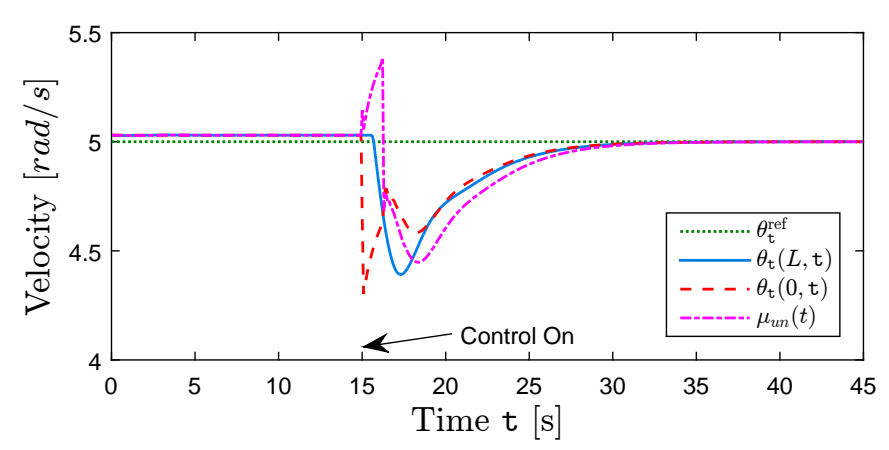

Fig. 8. Simulation of the top, bottom and delayed bottom estimated velocities, for $\lambda=0.9$.

In Figure 8, a high damping value is considered $(\lambda=0.9)$. Consequently, the open-loop system does not exhibit an oscillatory behavior. Therefore, the benefits of the feedback controller used after $15 s$ are quite reduced. However, this simulation highlights the robustness capabilities of this control law since the closed-loop convergence is still well-achieved.

\section{CONCLUSION}

We propose here a theoretical study of the mismatch robustness of an adaptive prediction-based control design. We wish to emphasize that the different ideas may be transposed to similar contexts. Finally, the interest of this result has been illustrated through simulations.

Future interesting works could include the study of robustness to other types of model mismatch, such as KelvinVoigt damping (a distributed term in $u_{x x t}$ ) which represents the dissipation within the matter.

Also, a future path to explore could be to develop control algorithms taking explicitly into account in-domain damping. A first step has been done in this sense in [20], but could be generalized by including adaptive control.

\section{APPENDIX A}

\section{INTERMEDIATE RESULTS}

\section{1) Relationship between transport equation and delay}

\section{Lemma 6: Consider}

$$
\begin{aligned}
& f_{t}(x, t)+c f_{x}(x, t)=\mathscr{F}(x, t) \\
& f(0, t)=\bar{f}(t) \text { if } c>0, \text { and } f(1, t)=\bar{f}(t) \text { if } c<0 \\
& f(., 0)=f_{0}
\end{aligned}
$$

in which $\mathscr{F} \in L_{2}((0, \infty) \times(0,1)), \bar{f} \in L_{2}(0, \infty), f_{0} \in L_{2}(0,1)$, and $c \in \mathbb{R} \backslash\{0\}$. There exists a unique weak solution $f \in L_{2}((0, \infty) \times(0,1))$ for the abstract Cauchy problem resulting from (117)-(119). This solution satisfies, for all $x \in[0,1]$, $t \in[0, \infty)$, and such that $0 \leqslant c(s-t)+x \leqslant 1$

$$
f(c(s-t)+x, s)=f(x, t)+\int_{t}^{s} \mathscr{F}(c(\tau-t)+x, \tau) d \tau
$$

Note that we can deduce for any $x$ the values of the distributed state $f(x, t)$ with the knowledge of its boundary and $\mathscr{F}$.

Proof: Without lack of generality, consider $c<0$ and the following variable

$$
m(x, t)=f(x, t)-\int_{0}^{x} \frac{1}{c} \mathscr{F}\left(s, t+\frac{s-x}{c}\right) d s
$$

One gets that, if $\mathscr{F} \in L_{2}((0, \infty) \times(0,1))$,

$$
m \in L_{2}((0, \infty) \times(0,1)) \Leftrightarrow f \in L_{2}((0, \infty) \times(0,1))
$$

Moreover, $m$ satisfies

$$
\begin{aligned}
& m_{t}(x, t)+c m_{x}(x, t)=0 \\
& m(1, t)=\bar{f}(t)-\int_{0}^{1} \frac{1}{c} \mathscr{F}\left(s, t+\frac{s-1}{c}\right) d s \\
& m(x, 0)=f_{0}(x)-\int_{0}^{x} \frac{1}{c} \mathscr{F}\left(s, \frac{s-x}{c}\right) d s
\end{aligned}
$$

which is a standard transport equation. Following [7] Example 2.2.4 or Exercise 3.14, this system is well-posed and its solution satisfies $m(x, t)=m\left(1, t-\frac{x}{c}\right)$ which, in turns, implies (120).

\section{2) Intermediate result for Lyapunov stability}

Proposition 5: Consider $x_{i} \in L_{2}(\mathbb{R}),(i \leqslant m, i, m \in \mathbb{N})$, and $V$ a positive definite functional of $\left(x_{i}^{2}\right)_{i=1 \ldots m}$. Assume there exists a increasing function $K$ such that

$$
\forall t \geqslant 0, \quad \dot{V}(t) \leqslant-\sum_{i=1}^{m}\left(a_{i}-K(V(t)) x_{i}(t)^{2}\right.
$$

in which $\forall i \in \llbracket 0, m \rrbracket, a_{i}>0$.

If $\forall i, a_{i}-K(V(0))>0$ then $\forall i, \forall t \geqslant 0, a_{i}-K(V(t))>0$

Proof : For the sake of simplicity, take $p=1$ (similar arguments hold for the general case). By contradiction, assume the existence of $t_{0}>0$ such that $a_{1}-K\left(V\left(t_{0}\right)\right) \leqslant 0$. By continuity, there exists at least one $t \in\left[0, t_{0}\right]$ such that $a_{1}-K(V(t))=0$. We denote $t_{1}>0$ the smallest. One has

$$
\forall t \in\left[0, t_{1}\left[, \quad a_{1}-K(V(t))>0\right.\right.
$$

and thus $V\left(t_{1}\right)<V(0)$ according to (126). Consequently, $a_{1}=$ $K\left(V\left(t_{1}\right)\right)<K(V(0))$ as $K$ is an increasing function, which is a contradiction.

\section{APPENDIX B}

\section{EXPRESSIONS OF $\dot{g}$ AND $\dot{W}$}

Proposition 6: The time derivative of $g$ (75), can be expressed as

$$
\begin{aligned}
& \dot{g}(t)=e^{a(q-1)}\left[a[\widehat{z}(0, t)+\widetilde{\delta}(0, t)+\mu(t) \widetilde{q}(t)]+\widetilde{d}_{\mu}(t)\right. \\
& \left.-\frac{a}{2}(a-1) \widetilde{\omega}(1, t)\right]+\left[e^{a(q-1)(1-\chi)}\left[\frac{\alpha(\chi / 2)}{2}+\widetilde{\delta}(\chi, t)\right]\right]_{\chi=0}^{1} \\
& +a \int_{0}^{1} e^{a(q-1)(1-\chi)} a(q-1)\left[\frac{\widehat{\alpha}(\chi / 2, t)}{2}+\widetilde{\delta}(\chi, t)\right] d \chi
\end{aligned}
$$

The time derivative of $W$ defined in (17) can be expressed as

$$
\begin{aligned}
\dot{W}(t) & =-\dot{\widehat{q}}(t)\left(e^{2 a(\widehat{q}(t)-1)} \mu(t)+2 a \int_{0}^{1} e^{2 a(\widehat{q}(t)-1)(1-\chi)} \widehat{\alpha}(\chi, t) d \chi\right) \\
& -\left(c_{0}+\widehat{q}(t)-1\right)\left(2 a \dot{\widehat{q}}(t) e^{2 a(\widehat{q}-1)} \mu(t)+e^{2 a(\widehat{q}(t)-1)}[a[\widehat{z}(0, t)\right. \\
& \left.+\widetilde{\delta}(0, t)+\mu(t) \widetilde{q}(t)]+\widetilde{d}_{\mu}(t)-\frac{a}{2}(q-1) \widetilde{\omega}(1, t)+\frac{\widetilde{\omega}_{t}(1, t)}{2}\right] \\
& +2 a \int_{0}^{1} e^{2 a(\widehat{q}(t)-1(1-\chi)}[2 a \dot{\widehat{q}}(t)(1-\chi) \widehat{\alpha}(\chi, t)-\hat{\widehat{d}}(t) \\
& \left.+a(\widehat{q}-1) \widehat{\alpha}(\chi, t)] d \chi+a\left[e^{2 a(\widehat{q}(t)-1(1-\chi)} \widehat{\alpha}(\chi, t)\right]_{\chi=0}^{1}\right)
\end{aligned}
$$


in which $\alpha$ can be expressed using the inverse backstepping transformation (72).

Proof: Taking the time-derivative of (76), using the transports (55) and (57), applying integration by part on $\widehat{\alpha}_{x}$ and $\tilde{\delta}_{x}$, and expressing $\tilde{d}$ with (73), one gets (128).

From the time derivative of (61), in a similar way, one gets (129).

\section{APPENDIX C \\ LYAPUNOV FUNCTIONAL ANALYSIS}

Before stating the proof of Lemma 2, one establishes the following proposition.

Proposition 7: There exist positive $M_{0}, M_{\omega}, M_{\omega t}, M_{i}\left(b_{1}\right), i \in$ $\{1,2, \ldots, 8\}, C_{i}, i \in\{1,2, \ldots, 8\}$ such that

$$
\begin{aligned}
\dot{V}_{1}(t) \leqslant & \frac{1}{1+N(t)}\left[-a c_{0} \mu(t)^{2}+\frac{4 a}{c_{0}} \widehat{z}(0, t)^{2}+\frac{4 a}{c_{0}} \widetilde{\delta}(0, t)^{2}\right. \\
& +\frac{a}{c_{0}}(q-1)^{2} \widetilde{\omega}(1, t)^{2}+\frac{1}{a c_{0}} \widetilde{\omega}_{t}(1, t)^{2} \\
& +b_{1}\left[-\widehat{z}(0, t)^{2}-\frac{\|\widehat{z}\|^{2}}{4}+\gamma_{q} M_{1}\left(b_{1}\right)\left(\mu(t)^{2}+\|\widehat{z}\|^{2}\right)\right. \\
& +\gamma_{d} M_{2}\left(b_{1}\right)\left(\mu(t)^{2}+\|\widehat{z}\|^{2}\right) \\
& \left.+M_{0} \widetilde{\delta}(0, t)^{2}+M_{\omega} \widetilde{\omega}(1, t)^{2}+M_{\omega t} \widetilde{\omega}_{t}(1, t)^{2}\right] \\
& +b_{2}\left[-\widehat{\beta}(1, t)^{2}+2 e\left(1+c_{0}+\widehat{q}(t)\right)^{2} \mu(t)^{2}+2 e \widehat{z}(0, t)^{2}\right. \\
& \left.\left.-\|\widehat{\beta}\|^{2}+\gamma_{d} M_{3}\left(b_{1}\right)\left(\mu(t)^{2}+\|\widehat{z}\|^{2}+\|\widehat{\beta}\|^{2}\right)\right]\right]
\end{aligned}
$$$$
\dot{V}_{2}(t) \leqslant b_{3}\left[-\widehat{\zeta}(0, t)^{2}-\|\widehat{\zeta}\|^{2}+C_{1}\left[\mu(t)^{2}+\|\widehat{z}\|^{2}\right]+\gamma_{d} M_{4}\left(b_{1}\right)\left[\mu(t)^{2}\right.\right.
$$$$
\left.\left.+\|\widehat{z}\|^{2}+\|\widehat{\zeta}\|^{2}\right]\right]+b_{4}\left[-\widehat{\omega}(1, t)^{2}-\|\widehat{\omega}\|^{2}+2 e \widehat{\zeta}(0, t)^{2}\right.
$$$$
+C_{2}\left[\mu(t)^{2}+\widetilde{d}_{\mu}(t)^{2}+\|\widetilde{z}\|^{2}+\|\widetilde{\delta}\|^{2}+\widetilde{\omega}(1, t)^{2}\right]
$$$$
\left.+\gamma_{d} M_{5}\left(b_{1}\right)\left[\mu(t)^{2}+\|\widehat{z}\|^{2}+\|\widehat{\omega}\|^{2}\right]\right]
$$

$$
\dot{V}_{3}(t) \leqslant b_{5}\left[-\widetilde{\zeta}(0, t)^{2}-(1+2 \lambda)\|\widetilde{\zeta}\|^{2}+\lambda C_{3}\left(\|\widetilde{\zeta}\|^{2}+\|\widehat{\zeta}\|^{2}+\|\widehat{\omega}\|^{2}\right.\right.
$$$$
\left.\left.+\|\widetilde{\omega}\|^{2}\right)\right]+b_{6}\left[-\widetilde{\omega}(1, t)^{2}+e \widetilde{\zeta}(0, t)^{2}-(1+2 \lambda)\|\widetilde{\omega}\|^{2}\right.
$$$$
\left.+\lambda C_{4}\left(\|\widetilde{\omega}\|^{2}+\|\widehat{\zeta}\|^{2}+\|\widehat{\omega}\|^{2}+\|\widetilde{\zeta}\|^{2}\right)\right]
$$$$
+b_{7}\left[e \widetilde{\zeta}(0, t)^{2}-\widetilde{\delta}(0, t)^{2}-\|\widetilde{\delta}\|^{2}\right]
$$

$$
\dot{V}_{5}(t) \leqslant b_{9}\left[-\widehat{\zeta}_{x}(0, t)^{2}-\left\|\widehat{\zeta}_{x}\right\|^{2}+C_{6}\left[\widetilde{\omega}_{t}(1, t)^{2}+\mu(t)^{2}+\widetilde{d}_{\mu}(t)^{2}\right.\right.
$$$$
\left.+\widehat{z}(0, t)^{2}+\|\widehat{z}\|^{2}+\widetilde{\delta}(0, t)^{2}+\widetilde{\omega}(1, t)^{2}\right]+\gamma_{d} M_{7}\left(b_{1}\right)\left[\mu(t)^{2}\right.
$$$$
\left.\left.+\|\widehat{z}\|^{2}\right]+\gamma_{q} M_{8}\left(b_{1}\right)\left[\mu(t)^{2}+\|\widehat{z}\|^{2}\right]\right]+b_{10}\left[-\widehat{\omega}_{x}(1, t)^{2}-\left\|\widehat{\omega}_{x}\right\|^{2}\right.
$$$$
+3 e \widehat{\zeta}_{x}(0, t)^{2}+C_{7}\left[\mu(t)^{2}+\widetilde{d}_{\mu}(t)^{2}+\widehat{z}(0, t)^{2}+\|\widehat{z}\|^{2}+\|\widetilde{\delta}\|^{2}\right.
$$$$
\left.\left.+\widetilde{\delta}(0, t)^{2}+\widetilde{\omega}(1, t)^{2}+\widetilde{\zeta}(0, t)^{2}\right]\right]+b_{11}\left[-\widetilde{\zeta}_{t}(0, t)^{2}\right.
$$$$
\left.-(1+2 \lambda)\left\|\widetilde{\zeta}_{t}\right\|^{2}+\lambda C_{8}\left(\left\|\widetilde{\zeta}_{t}\right\|^{2}+\left\|\widehat{\zeta}_{x}\right\|^{2}+\left\|\widehat{\omega}_{x}\right\|^{2}+\left\|\widetilde{\omega}_{t}\right\|^{2}\right)\right]
$$$$
+b_{12}\left[-\widetilde{\omega}_{t}(1, t)^{2}-(1+2 \lambda)\left\|\widetilde{\omega}_{t}\right\|^{2}+e \widetilde{\zeta}_{t}(0, t)^{2}\right.
$$$$
\left.+\lambda C_{9}\left(\left\|\widetilde{\omega}_{t}\right\|^{2}+\left\|\widehat{\zeta}_{x}\right\|^{2}+\left\|\widehat{\omega}_{x}\right\|^{2}+\left\|\widetilde{\zeta}_{t}\right\|^{2}\right)\right]
$$

$$
\begin{aligned}
\dot{V}_{4}(t) & \leqslant b_{8}\left(-a c_{0} \widetilde{d}_{\mu}(t)^{2}+\gamma_{d} M_{6}\left(b_{1}\right)\left[\mu(t)^{2}+\|\widehat{z}\|^{2}\right]\right. \\
& \left.+C_{5}\left[\mu^{2}+\widehat{z}(0, t)^{2}+\widetilde{\delta}(0, t)^{2}+\widetilde{\omega}(1, t)^{2}+\widetilde{\omega}_{t}(1, t)^{2}\right]\right)
\end{aligned}
$$

Proof : For simplicity, we only detail the proof of (130), as the remaining inequalities can be obtained with similar arguments. From the definition of $V_{1}(t)$ one gets,

$$
\dot{V}_{1}(t)=\frac{\dot{N}(t)}{1+N(t)}-2 \frac{\widetilde{q}(t) \dot{\hat{q}}(t)}{\gamma_{q}}-2 \frac{\widetilde{d}(t) \dot{\hat{d}(t)}}{\gamma_{d}}
$$

From the expression of $N(89)$, it follows that

$$
\dot{N}(t)=2 \dot{\mu}(t) \mu(t)+4 b_{1} \int_{0}^{1} e^{x} \widehat{z}_{t} d x+2 b_{2} \int_{0}^{1} e^{1-x} \widehat{\beta}_{3} \widehat{\beta}_{t} d x
$$

Then, using (64), and Young inequality, one obtains

$$
\begin{aligned}
& 2 \dot{\mu}(t) \mu(t) \leqslant-a c_{0} \mu(t)^{2}+\frac{4 a}{c_{0}} \widehat{z}(0, t)^{2}+\frac{4 a}{c_{0}} \widetilde{\delta}(0, t)^{2}+\frac{a}{c_{0}}(q-1)^{2} \\
& \times \widetilde{\omega}(1, t)^{2}+\frac{1}{a c_{0}} \widetilde{\omega}_{t}(1, t)^{2}+2 a \mu(t)(\mu(t) \widetilde{q}(t)-\widetilde{d}(t))
\end{aligned}
$$

Finally using (65)-(68), and Young and Cauchy-Schwarz inequalities, with integrations by parts, one obtains

$$
\begin{gathered}
4 \int_{0}^{1} e^{x} \widehat{z}_{z_{t}} d x \leqslant-\widehat{z}(0, t)^{2}-\frac{\|\widehat{z}\|^{2}}{4}+\gamma_{q} M_{1}\left(b_{1}\right)\left(\mu(t)^{2}+\|\widehat{z}\|^{2}\right) \\
+\gamma_{d} M_{2}\left(b_{1}\right)\left(\mu(t)^{2}+\|\widehat{z}\|^{2}\right)+M_{0} \widetilde{\delta}(0, t)^{2}+M_{\omega} \widetilde{\omega}(1, t)^{2} \\
\left.+M_{\omega t} \widetilde{\omega}_{t}(1, t)^{2}\right]+2 a\left(c_{0}+\widehat{q}(t)-1\right) \\
\quad \times \int_{0}^{1} e^{2\left(a(\widehat{q}(t)-1)+\frac{1}{2}\right) x \widehat{z}(x, t)[\mu(t) \widetilde{q}(t)-\widetilde{d}(t)] d x} \\
2 \int_{0}^{1} e^{1-x} \widehat{\beta} \widehat{\beta}_{t} d x \leqslant-\widehat{\beta}(1, t)^{2}+2 e\left(1+c_{0}+\widehat{q}(t)\right)^{2} \mu(t)^{2} \\
\quad+2 e \widehat{z}(0, t)^{2}-\|\widehat{\beta}\|^{2}+\gamma_{d} M_{3}\left(b_{1}\right)\left(\mu(t)^{2}+\|\widehat{z}\|^{2}+\|\widehat{\beta}\|^{2}\right)
\end{gathered}
$$

Gathering (135)-(139), and using Claim 2 concludes the proof of (130).

Proof of Lemma 2: Gathering inequalities (130)-(133) of Proposition 7, we obtain

$$
\dot{V}\left(\mathscr{X}_{e}(t)\right) \leqslant \sum_{\mathscr{V} \in \mathscr{S}} \delta_{\mathscr{V}} f_{\mathscr{V}}(.) \mathscr{V}^{2}
$$

in which

$$
\begin{gathered}
\mathscr{S}=\{\mu,\|\vec{z}\|,\|\widehat{\beta}\|,\|\widetilde{\delta}\|,\|\widehat{\zeta}\|,\|\widehat{\omega}\|,\|\widetilde{\zeta}\|,\|\widetilde{\omega}\|, \\
\widetilde{d}_{\mu},\left\|\widehat{\zeta}_{x}\right\|,\left\|\widehat{\omega}_{x}\right\|,\left\|\widetilde{\zeta}_{t}\right\|,\left\|\widetilde{\omega}_{t}\right\|, \widehat{z}|0, \widehat{\beta}| 1, \widetilde{\delta} \mid 0 \\
\left.\widehat{\zeta}|0, \widehat{\omega}| 1, \widetilde{\zeta}|0, \widetilde{\omega}| 1, \widehat{\zeta}_{x}\left|0, \widehat{\omega}_{x}\right| 1, \widetilde{\zeta}_{t}\left|0, \widetilde{\omega}_{t}\right| 1\right\}
\end{gathered}
$$

where $\widehat{\omega} \mid 1$ denote the boundary of $\widehat{\omega}$ for $x=1$, i.e., $\widehat{\omega}(1, t)$

$$
\delta_{\mathscr{V}}=\left\{\begin{array}{l}
\frac{1}{1+N(t)}, \quad \text { if } \quad \mathscr{V} \in\{z|0, \mu,\|\widehat{z}\|,\|\widehat{\beta}\|, \widehat{\beta}| 1\} \\
1
\end{array}\right.
$$


and with

$$
\begin{aligned}
& f_{\bar{z} \mid 0}\left(b_{i}, V(t)\right)=\frac{4 a}{c_{0}}-b_{1}+2 e b_{2}+\left[b_{8} C_{5}+b_{9} C_{6}+b_{10} C_{7}\right] e^{V(t)} \\
& f_{\widetilde{\delta} \mid 0}\left(b_{i}\right)=\frac{4 a}{c_{0}}+b_{1} M_{0}-b_{7}+b_{8} C_{5}+b_{9} C_{6}+b_{10} C_{7} \\
& f_{\mu}\left(b_{i}, V(t)\right)=-a c_{0}+b_{1} \gamma_{q} M_{1}+b_{1} \gamma_{d} M_{2}+2 e b_{2}\left(1+c_{0}+\bar{q}\right)^{2} \\
& +b_{2} \gamma_{d} M_{3}+e^{V(t)}\left[b_{3} C_{1}+b_{3} \gamma_{d} M_{4}+b_{4} C_{2}+b_{4} \gamma_{d} M_{5}\right. \\
& +b_{8} \gamma_{d} M_{6}+b_{8} C_{5}+b_{9} C_{6}+b_{9} \gamma_{q} M_{7}+b_{9} \gamma_{d} M_{8} \\
& \left.+b_{10} C_{7}\right] \\
& f_{\widetilde{d}_{\mu}}\left(b_{i}\right)=b_{4} C_{2}-a c_{0} b_{8}+b_{9} C_{6}+b_{10} C_{7} \\
& f_{\widehat{\zeta} \mid 0}\left(b_{i}\right)=-b_{3}+2 e b_{4} \\
& f_{\widetilde{\zeta} \mid 0}\left(b_{i}\right)=-b_{5}+e b_{6}+e b_{7}+b_{10} C_{7} \\
& f_{\widetilde{\omega} \mid 1}\left(b_{i}\right)=\frac{a}{c_{0}}(q-1)^{2}+b_{1} M_{\omega}+b_{4} C_{2}-b_{6} \\
& +b_{8} C_{5}+b_{9} C_{6}+b_{10} C_{7} \\
& f_{\|\vec{z}\|}\left(b_{i}, V(t)\right)=-\frac{b_{1}}{4}+b_{1} \gamma_{q} M_{1}+b_{1} \gamma_{d} M_{2}+b_{2} \gamma_{d} M_{3}+e^{V(t)}\left[b_{3} C_{1}\right. \\
& +b_{3} \gamma_{d} M_{4}+b_{4} C_{2}+b_{4} \gamma_{d} M_{5}+b_{8} \gamma_{d} M_{6}+b_{9} C_{6} \\
& \left.+b_{9} \gamma_{q} M_{7}+b_{9} \gamma_{d} M_{8}+b_{10} C_{7}\right] \\
& f_{\|\widehat{\beta}\|}\left(b_{i}\right)=-b_{2}+b_{2} \gamma_{d} M_{3} \\
& f_{\|\widehat{\zeta}\|}\left(b_{i}, \lambda\right)=-b_{3}+b_{3} \gamma_{d} M_{4}+b_{5} \lambda C_{3}+b_{6} \lambda C_{4} \\
& f_{\|\widetilde{\zeta}\|}\left(b_{i}, \lambda\right)=-b_{5}(1+2 \lambda)+b_{5} \lambda C_{3}+b_{6} \lambda C_{4} \\
& f_{\|\widehat{\omega}\|}\left(b_{i}, \lambda\right)=-b_{4}+b_{4} \gamma_{d} M_{5}+b_{5} \lambda C_{3}+b_{6} \lambda C_{4} \\
& f_{\|\widetilde{\omega}\|}\left(b_{i}, \lambda\right)=-b_{6}(1+2 \lambda)+b_{5} \lambda C_{3}+b_{6} \lambda C_{4} \\
& f_{\|\widetilde{\delta}\|}\left(b_{i}\right)=b_{4} C_{2}-b_{7}+b_{10} C_{7} \\
& f_{\widehat{\zeta}_{x} \mid 0}\left(b_{i}\right)=-b_{9}+3 e b_{10} \\
& f_{\widetilde{\zeta}_{t} \mid 0}\left(b_{i}\right)=-b_{11}+e b_{12} \\
& f_{\widetilde{\omega}_{t} \mid 1}\left(b_{i}\right)=\frac{1}{a c_{0}}+b_{1} M_{\omega t}+b_{8} C_{5}+b_{9} C_{6}-b_{12} \\
& f_{\left\|\widehat{\zeta}_{x}\right\|}\left(b_{i}, \lambda\right)=-b_{9}+b_{11} \lambda C_{8}+b_{12} \lambda C_{9} \\
& f_{\left\|\widetilde{\zeta}_{t}\right\|}\left(b_{i}, \lambda\right)=-b_{11}(1+2 \lambda)+b_{11} \lambda C_{8}+b_{12} \lambda C_{9} \\
& f_{\left\|\widehat{\omega}_{x}\right\|}\left(b_{i}, \lambda\right)=-b_{10}+b_{11} \lambda C_{8}+b_{12} \lambda C_{9} \\
& f_{\left\|\widetilde{\omega}_{t}\right\|}\left(b_{i}, \lambda\right)=-b_{12}(1+2 \lambda)+b_{11} \lambda C_{8}+b_{12} \lambda C_{9}
\end{aligned}
$$

Note that, we used that $1+N(t) \leqslant e^{V(t)}$ (from the definition of $V(90))$.

A sufficient condition for the stability of the Extended Target system of state $\mathscr{X}_{e}(86)$ is therefore the existence of parameters $b_{i}, \gamma_{q}, \gamma_{d}$, and $\lambda$ such that

$$
f_{\mathscr{V}}(.)<0, \mathscr{V} \in \mathscr{S}
$$

This is the condition we investigate in the following.

With this aim in view, we follow the following procedure to select the different parameters

$$
\begin{aligned}
b_{2} & \rightarrow b_{1} \rightarrow\left(b_{6}, b_{7}, b_{12}\right) \rightarrow\left(b_{11}, b_{5}\right) \\
\quad \rightarrow\left(\gamma_{q}, \gamma_{d}\right) & \rightarrow\left(b_{3}, b_{8}\right) \rightarrow\left(b_{9}, b_{4}\right) \rightarrow b_{10}
\end{aligned}
$$

First, from (143) and (145) we choose $b_{2}$ and $b_{1}$ such that

$$
\begin{gathered}
b_{2}<\frac{a c_{0}}{2 e\left(1+c_{0}+\bar{q}\right)^{2}} \\
b_{1}>\frac{4 a}{c_{0}}+2 e b_{2}
\end{gathered}
$$

Then from (144), (149), (159) we choose respectively $b_{7}, b_{6}$, and $b_{12}$ such that

$$
\begin{aligned}
& b_{7}>\frac{4 a}{c_{0}}+b_{1} M_{0} \\
& b_{6}>\frac{a}{c_{0}}(\bar{q}-1)^{2}+b_{1} M_{\omega} \\
& b_{12}>\frac{1}{a c_{0}}+b_{1} M_{\omega t}
\end{aligned}
$$

$b_{11}$ is taken, according to (157), such that

$$
b_{11}>e b_{12}
$$

and from (148) we choose $b_{5}$ as

$$
b_{5}>e\left(b_{6}+b_{7}\right)
$$

$\gamma_{q}$ and $\gamma_{d}$ are chosen, according to (145), (150), (151), (152), and (154), as

$$
\begin{aligned}
& \gamma_{q}+\gamma_{d}<\min \left\{\frac{a c_{0}-2 e b_{2}\left(1+c_{0}+\bar{q}\right)^{2}}{b_{1} M_{1}+b_{1} M_{2}+b_{2} M_{3}},\right. \\
& \left.\frac{b_{1}}{4\left(b_{1} M_{1}+b_{1} M_{2}+b_{2} M_{3}\right)}, \frac{1}{M_{3}}, \frac{1}{M_{4}}, \frac{1}{M_{5}}\right\}
\end{aligned}
$$

Note that, if $b_{i}<1, i \in\{3,4,8,9,10\}$, this implies the existence of $M\left(\mathscr{X}_{e}(0)\right)>0$ independent of $b_{i}, i \in\{3,4,8,9,10\}$, such that $V\left(\mathscr{X}_{e}(0)\right) \leqslant M\left(\mathscr{X}_{e}(0)\right)$ which is simply denoted $M$ in the following. Consequently, the parameter $b_{3}$ is fixed, according to (145) and (150), as ${ }^{1}$

$$
\begin{gathered}
b_{3}<\min \left\{1, \frac{-f_{\mu}\left(b_{3,4,8,9,10}=0, M\right)}{e^{M}\left[C_{1}+\gamma_{d} M_{4}\right]},\right. \\
\left.\frac{-f_{\|\mid \hat{z}\|}\left(b_{3,4,8,9,10}=0, M\right)}{e^{M}\left[C_{1}+\gamma_{d} M_{4}\right]}\right\}
\end{gathered}
$$

Then, $b_{8}$ is taken according to (143)-(145), (149), (150), and (159), as

$$
\begin{aligned}
& b_{8}<\min \left\{1, \frac{-f_{\widetilde{z} \mid 0}\left(b_{4,8,9,10}=0, M\right)}{e^{M} C_{5}}, \frac{-f_{\widetilde{\delta} \mid 0}\left(b_{4,8,9,10}=0\right)}{e^{M} C_{5}},\right. \\
& \frac{-f_{\mu}\left(b_{4,8,9,10}=0, M\right)}{e^{M}\left[\gamma_{d} M_{6}+C_{5}\right]}, \frac{-f_{\widetilde{\omega} \mid 1}\left(b_{4,8,9,10}=0\right)}{C_{5}}, \\
& \left.\frac{-f_{\|z\|}\left(b_{4,8,9,10}=0, M\right)}{e^{M} \gamma_{d} M_{6}}, \frac{-f_{\widetilde{\omega}_{t} \mid 1}\left(b_{4,8,9,10}=0\right)}{C_{5}}\right\}
\end{aligned}
$$

From (143)-(146), (149), (150) and (159), $b_{9}$ is fixed as

$$
\begin{aligned}
& b_{9}<\min \left\{1, \frac{-f_{\tilde{z} \mid 0}\left(b_{4,9,10}=0, M\right)}{C_{6} e^{M}}, \frac{-f_{\widetilde{\delta} \mid 0}\left(b_{4,9,10}\right)}{C_{6} e^{M}},\right. \\
& \frac{-f_{\mu}\left(b_{4,9,10}=0, M\right)}{e^{M}\left[C_{6}+\gamma_{q} M_{7}+\gamma_{d} M_{8}\right]}, \frac{-f_{\widetilde{d_{\mu}}}\left(b_{4,9,10}=0\right)}{C_{6}}, \frac{-f_{\widetilde{\omega} \mid 1}\left(b_{4,9,10}=0\right)}{C_{6}}, \\
& \left.\frac{-f_{\|z\|}\left(b_{4,9,10}=0, M\right)}{e^{M}\left[C_{6}+\gamma_{q} M_{7}+\gamma_{d} M_{8}\right]}, \frac{-f_{\widetilde{\omega}_{t} \mid 1}\left(b_{4,9,10}=0\right)}{C_{6}}\right\}
\end{aligned}
$$

${ }^{1}$ By $f_{\mu}\left(b_{3,4,8,9,10}=0, M\right)$ we mean $f_{\mu}\left(b_{1}, b_{2}, b_{3}=0, b_{4}=0, b_{5}, b_{6}, b_{7}, b_{8}=\right.$ $\left.\left.0, b_{9}=0, b_{10}=0, b_{11}, b_{12}, V=M\right)\right)$. 
From (145)-(146), (147), (149), (150), and (156), $b_{4}$ is chosen with respect to

$$
\begin{aligned}
b_{4}< & \min \left\{1, \frac{-f_{\mu}\left(b_{4,10}=0, M\right)}{C_{2}+\gamma_{d} M_{5}}, \frac{-f_{\widetilde{d_{\mu}}}\left(b_{4,10}=0\right)}{C_{2}}, \frac{b_{3}}{2 e},\right. \\
& \left.\frac{-f_{\widetilde{\omega} \mid 1}\left(b_{4,10}=0\right)}{C_{2}}, \frac{b_{7}}{C_{2}}, \frac{-f_{\|\bar{z}\|}\left(b_{4,10}=0, M\right)}{C_{2}+\gamma_{d} M_{5}}\right\}
\end{aligned}
$$

The parameters $b_{10}$ is fixed according to (143)-(146), (148), (149), (150), (156), (157), and (159) as

$$
\begin{aligned}
& b_{10}<\min \left\{1, \frac{-f_{\widetilde{z} \mid 0}\left(b_{10}=0, M\right)}{C_{7} e^{M}}, \frac{f_{\widetilde{\delta} \mid 0}\left(b_{10}=0\right)}{C_{7} e^{M}}, \frac{-f_{\mu}\left(b_{10}=0, M\right)}{C_{7} e^{M}},\right. \\
& \frac{-f_{\widetilde{d}_{\mu}}\left(b_{10}=0\right)}{C_{7}}, \frac{b_{5}-e b_{6}-e b_{7}}{C_{7}}, \frac{-f_{\widetilde{\omega} \mid 1}\left(b_{10}=0\right)}{C_{7}}, \frac{b_{7}-b_{4} C_{2}}{C_{7}} \\
& \left.\frac{-f_{\|\mid \bar{z}\|}\left(b_{10}=0, M\right)}{C_{7} e^{M}}, \frac{b_{9}}{3 e}\right\}
\end{aligned}
$$

Finally, a upper bound for $\lambda$ is found according to (152)(155), and (160)-(163) as

$$
\begin{aligned}
& \lambda<\min \left\{\frac{b_{3}\left(1-\gamma_{q} M_{4}\right)}{b_{5} C_{3}+b_{6} C_{4}}, \frac{b_{5}}{b_{5}\left(C_{3}-2\right)+b_{6} C_{4}},\right. \\
& \frac{b_{4}-b_{4} \gamma_{q} M_{5}}{b_{5} C_{3}+b_{6} C_{4}}, \frac{b_{6}}{b_{5} C_{3}+b_{6} C_{4}-2 b_{6}} \\
& \frac{b_{9}}{b_{11} C_{8}+b_{12} C_{9}}, \frac{b_{11}}{b_{11}\left(C_{8}-2\right)+b_{12} C_{9}}, \\
& \left.\frac{b_{10}}{b_{11} C_{8}+b_{12} C_{9}}, \frac{b_{12}}{b_{11} C_{8}+b_{12}\left(C_{9}-2\right)}\right\}
\end{aligned}
$$

by assuming that $C_{3}>2, C_{4}>2, C_{8}>2$ and $C_{9}>2$, which can be taken as such.

Using Proposition 5 in Appendix A-2, with this choice of parameters, one obtains the existence of $\vartheta>0$ such that $f_{\mathscr{V}}()<.-\vartheta$ for all $\mathscr{V} \in \mathscr{S}$. From (164), this implies that (96) holds which concludes the proof of Lemma 2.

\section{APPENDIX D}

PROOFS OF LEMMAS 3 AND 4

Proof of Lemma 3: We start by observing that

$$
\begin{aligned}
& u_{t}(0, \tau)=e^{a(q-1)(\tau-t-1)}\left(\mu(t)-\frac{1}{2} \widetilde{\omega}(1, t)\right) \\
& +2 a \int_{0}^{\tau+1-t} e^{a(q-1)(\tau+1-t-\chi)}[\widehat{\alpha}(\chi / 2, t)+\widetilde{\delta}(\chi, t)-\widetilde{d}(t)] d \chi
\end{aligned}
$$

which can be obtained by arguments similar to those used to prove (76).

Then, using the fact that

$$
\begin{aligned}
& \widetilde{\omega}(1, t)=\int_{0}^{1} \widetilde{\omega}_{x}(\chi, t) d \chi+\widetilde{\omega}(0, t) \\
& \widetilde{\zeta}(0, t)=-\int_{0}^{1} \widetilde{\zeta}_{x}(\chi, t) d \chi+\widetilde{\zeta}(1, t)
\end{aligned}
$$

together with (44) and (74), $\widetilde{\omega}(1, t)$ can be expressed as

$$
\widetilde{\omega}(1, t)=\int_{0}^{1}\left[\widetilde{\zeta}_{t}(\chi, t)-\widetilde{\omega}_{t}(\chi, t)\right] d \chi
$$

which implies, with Young's and Cauchy-Schwarz's inequality, that

$$
\widetilde{\omega}(1, t)^{2} \leqslant 2\left(\left\|\widetilde{\zeta}_{t}\right\|^{2}+\left\|\widetilde{\omega}_{t}\right\|^{2}\right)
$$

Therefore, from (179) and (183), and using Young inequality on (72), there exists $C_{1}>0$ such that

$$
\begin{aligned}
u_{t}(0, t)^{2} \leqslant & \mathrm{C}_{1}\left[\mu(t)^{2}+\|\widetilde{z}\|^{2}+\|\widetilde{\delta}\|^{2}+\widetilde{d}(t)^{2}\right. \\
& \left.+\left\|\widetilde{\zeta}_{t}\right\|^{2}+\left\|\widetilde{\omega}_{t}\right\|^{2}\right]
\end{aligned}
$$

which gives the existence of $r_{1}>0$ satisfying

$$
u_{t}(0, t)^{2} \leqslant r_{1}\left(e^{V(t)}-1\right)
$$

Furthermore, from the definition of the Riemann variables, (15)-(16) and (37)-(38), one gets

$$
\begin{aligned}
& u_{t}(x, t)=\frac{\widehat{\zeta}(x, t)+\widehat{\omega}(x, t)+\widetilde{\zeta}(x, t)+\widetilde{\omega}(x, t)}{2} \\
& u_{x}(x, t)-\widehat{d}(t)=\frac{\widehat{\zeta}(x, t)-\widehat{\omega}(x, t)+\widetilde{\zeta}(x, t)-\widetilde{\omega}(x, t)}{2} \\
& u_{x t}(x, t)=\frac{\widehat{\zeta}_{x}(x, t)+\widehat{\omega}_{x}(x, t)+\widetilde{\zeta}_{t}(x, t)-\widetilde{\omega}_{t}(x, t)}{2} \\
& u_{x x}(x, t)=\frac{\widehat{\zeta}_{x}(x, t)-\widehat{\omega}_{x}(x, t)+\widetilde{\zeta}_{t}(x, t)+\widetilde{\omega}_{t}(x, t)}{2} \\
&+\lambda(\widehat{\zeta}+\widehat{\omega}+\widetilde{\zeta}+\widetilde{\omega})(x, t)
\end{aligned}
$$

and, applying Young's, Cauchy-Schwarz's inequalities, one can get

$$
\begin{aligned}
\left\|u_{t}\right\|^{2} & \leqslant\|\widehat{\zeta}\|^{2}+\|\widehat{\omega}\|^{2}+\|\widetilde{\zeta}\|^{2}+\|\widetilde{\omega}\|^{2} \\
\left\|u_{x}-d\right\|^{2} & \leqslant \frac{5}{4}\left(\|\widehat{\zeta}\|^{2}+\|\widehat{\omega}\|^{2}+\|\widetilde{\zeta}\|^{2}+\|\widetilde{\omega}\|^{2}\right)+5 \widetilde{d}(t)^{2} \\
\left\|u_{t x}\right\|^{2} & \leqslant\left\|\widehat{\zeta}_{x}\right\|^{2}+\left\|\widehat{\omega}_{x}\right\|^{2}+\left\|\widetilde{\zeta}_{t}\right\|^{2}+\left\|\widetilde{\omega}_{t}\right\|^{2} \\
\left\|u_{x x}\right\|^{2} & \leqslant 4\left[\left\|\widehat{\zeta}_{x}\right\|^{2}+\left\|\widehat{\omega}_{x}\right\|^{2}+\left\|\widetilde{\zeta}_{t}\right\|^{2}+\left\|\widetilde{\omega}_{t}\right\|^{2}\right] \\
& +8 \lambda^{2}\left[\|\widehat{\zeta}\|^{2}+\|\widehat{\omega}\|^{2}+\|\widetilde{\zeta}\|^{2}+\|\widetilde{\omega}\|^{2}\right]
\end{aligned}
$$

Therefore, from (190)-(193)

$$
\begin{aligned}
\left\|u_{t}\right\|^{2}+\left\|u_{x}-d\right\|^{2} & +\left\|u_{t x}\right\|^{2}+\left\|u_{x x}\right\|^{2} \\
& \leqslant\left(r_{2}+5 \gamma_{d}\right)\left(e^{V(t)}-1\right)
\end{aligned}
$$

for a given $r_{2}>0$. Finally

$$
\widetilde{d}(t)^{2}+\widetilde{q}(t)^{2} \leqslant\left(\gamma_{d}+\gamma_{d}\right) V(t)
$$

Consequently, gathering (185), (194)-(195), we obtain (98). This concludes the proof of Lemma 3.

Proof of Lemma 4: From the definition of $V$ (90), one obtains

$$
\begin{aligned}
V(t) \leqslant & \mu(t)^{2}+2 e b_{1}\|\widehat{z}\|^{2}+e b_{2}\|\widehat{\beta}\|+e b_{3}\|\widehat{\zeta}\|^{2}+e b_{4}\|\widehat{\omega}\|^{2} \\
& +e b_{5}\|\widetilde{\zeta}\|^{2}+e b_{6}\|\widetilde{\omega}\|^{2}+e b_{7}\|\widetilde{\delta}\|^{2}+b_{8} \widetilde{d}_{\mu}(t)^{2} \\
& +e b_{9}\left\|\widehat{\zeta}_{x}\right\|^{2}+e b_{10}\left\|\widehat{\omega}_{x}\right\|^{2}+e b_{11}\left\|\widetilde{\zeta}_{t}\right\|^{2}+e b_{12}\left\|\widetilde{\omega}_{t}\right\|^{2}
\end{aligned}
$$

From (59), using (183), it holds

$$
\mu(t)^{2} \leqslant 2 u_{t}(0, t-1)^{2}+\left\|\widetilde{\zeta}_{t}\right\|^{2}+\left\|\widetilde{\omega}_{t}\right\|^{2}
$$

Then, from the backstepping transformation (63) and from the definition of $\widehat{\alpha}(51)$, there exists $\mathrm{C}_{2}>0$ such that

$$
\|\widehat{z}\| \leqslant \mathrm{C}_{2}\left[\mu(t)^{2}+\max _{s \in[0,1]}\left[\|\widehat{\zeta}(t-s)\|^{2}+\widetilde{d}(t-s)^{2}\right]\right]
$$


Besides, according to the definition of $\widehat{\beta}$ (49), one obtains the existence of $\mathrm{C}_{3}>0$

$$
\begin{aligned}
\|\widehat{\beta}\|^{2} \leqslant & \mathrm{C}_{3}\left[\|\widehat{\omega}(t-1)\|^{2}+\max _{s \in[0,1]}\left[\tilde{d}(t-s)^{2}\right.\right. \\
& \left.\left.+\left\|\widetilde{\zeta}_{t}(t-s)\right\|^{2}+\left\|\widetilde{\omega}_{t}(t-s)\right\|^{2}\right]\right]
\end{aligned}
$$

Furthermore, from the definition of $\widetilde{d}_{\mu}$ (73) one writes

$$
\exists \mathrm{C}_{4}>0, \quad \widetilde{d}_{\mu}(t)^{2} \leqslant \mathrm{C}_{4}\left[\mu(t)^{2}+\widetilde{d}(t)^{2}\right]
$$

Finally, by the definition of $\widetilde{\zeta}, \widetilde{\omega}, \widehat{\zeta}$, and $\widehat{\omega},(35)-(38)$ and using the expression (186), one gets

$$
\begin{aligned}
\widetilde{\zeta}(x, t)= & -2 \lambda \int_{x}^{1} u_{t}(\chi, t+x-\chi) d \chi \\
\widetilde{\omega}(x, t)= & -2 \lambda \int_{0}^{x} u_{t}(\chi, t-x+\chi) d \chi \\
& +2 \lambda \int_{0}^{1} u_{t}(\chi, t-x-\chi) d \chi \\
\widehat{\zeta}(x, t)= & u_{t}(x, t)+u_{x}(x, t)-\widehat{d}(t)-\widetilde{\zeta}(x, t) \\
\widehat{\omega}(x, t)= & u_{t}(x, t)-u_{x}(x, t)+\widehat{d}(t)-\widetilde{\omega}(x, t)
\end{aligned}
$$

and concludes, applying Cauchy-Schwarz's inequality, that

$$
\begin{aligned}
\|\widetilde{\zeta}(t)\|^{2} \leqslant & 4 \lambda^{2} \max _{s \in[0,1]}\left\|u_{t}(t-s)\right\|^{2} \\
\|\widetilde{\omega}(t)\|^{2} \leqslant & 8 \lambda^{2} \max _{s \in[0,2]}\left\|u_{t}(t-s)\right\|^{2} \\
\|\widehat{\zeta}(t)\|^{2} \leqslant & 4\left(\left\|u_{t}(t)\right\|^{2}+\left\|u_{x}(t)-d\right\|^{2}+\widetilde{d}(t)^{2}\right. \\
& \left.+4 \lambda^{2} \max _{s \in[0,1]}\left\|u_{t}(t-s)\right\|^{2}\right) \\
\|\widehat{\omega}(t)\|^{2} \leqslant & 4\left(\left\|u_{t}(t)\right\|^{2}+\left\|u_{x}(t)-d\right\|+\widetilde{d}(t)^{2}\right. \\
& \left.+8 \lambda^{2} \max _{s \in[0,2]}\left\|u_{t}(t-s)\right\|^{2}\right)
\end{aligned}
$$

Similarly, there exist positive constants $\mathrm{C}_{5}, \mathrm{C}_{6}, \mathrm{C}_{7}, \mathrm{C}_{8}$, and $\mathrm{C}_{9}$ such that

$$
\begin{aligned}
& \|\widetilde{\delta}\|^{2} \leqslant \mathrm{C}_{5} \max _{s \in[0,2]}\left\|u_{t}(t-s)\right\|^{2} \\
& \left\|\widetilde{\zeta}_{t}\right\|^{2} \leqslant \mathrm{C}_{6} \max _{s \in[0,1]}\left[\left\|u_{x x}(t-s)\right\|^{2}+\left\|u_{t}(t-s)\right\|^{2}\right] \\
& \left\|\widetilde{\omega}_{t}\right\|^{2} \leqslant \mathrm{C}_{7} \max _{s \in[0,2]}\left[\left\|u_{x x}(t-s)\right\|^{2}+\left\|u_{t}(t-s)\right\|^{2}\right] \\
& \left\|\widehat{\omega}_{x}\right\|^{2} \leqslant \mathrm{C}_{8}\left(\left\|u_{t x}\right\|^{2}+\max _{s \in[0,2]}\left[\left\|u_{x x}(t-s)\right\|^{2}+\left\|u_{t}(t-s)\right\|^{2}\right]\right) \\
& \left\|\widehat{\zeta}_{x}\right\|^{2} \leqslant \mathrm{C}_{9}\left(\left\|u_{t x}\right\|^{2}+\max _{s \in[0,2]}\left[\left\|u_{x x}(t-s)\right\|^{2}+\left\|u_{t}(t-s)\right\|^{2}\right]\right)
\end{aligned}
$$

Thus, gathering inequalities (196)-(200), and (205)-(213), it holds for a suitable $\rho>0$

$$
V(t) \leqslant \rho \max _{s \in[0,3]} \Gamma\left(\mathscr{X}_{s}(t-s)\right)
$$

This concludes the proof of Lemma 4.

\section{APPENDIX E}

\section{PROOF OF LEMMA 5}

From (97), one can easily get that $N(t), \widetilde{q}(t), \widetilde{d}(t)$, and $V_{2}(t)$, $V_{3}(t)$ and $V_{4}(t)$ are uniformly bounded for $t \geqslant 0$. Therefore, $\mu(t),\|\widehat{z}(t)\|,\|\widehat{\beta}(t)\|,\|\widehat{\omega}(t)\|,\|\widehat{\zeta}(t)\|,\|\widetilde{\zeta}(t)\|,\|\widetilde{\omega}(t)\|,\|\widetilde{\delta}(t)\|$, $\widetilde{d}_{\mu}(t),\left\|\widehat{\omega}_{x}(t)\right\|,\left\|\widehat{\zeta}_{x}(t)\right\|,\left\|\widetilde{\zeta}_{t}(t)\right\|$ and $\left\|\widetilde{\omega}_{t}(t)\right\|$ are also uniformly bounded for $t \geqslant 0$.

From there, applying Young's inequality to (9) and (10), one can obtain that $\dot{\hat{q}}(t)$ and $\dot{\hat{d}}(t)$ are uniformly bounded for $t \geqslant 0$. Similarly, applying Cauchy-Schwarz's inequality to (6), one can obtain that $\widehat{\zeta}(1, t)$ and thus $\widehat{\alpha}(1, t)$ are uniformly bounded for $t \geqslant 0$. Moreover, using Lemma 6 in Appendix A-1, $\widehat{\zeta}(x, t)$ is also uniformly bounded for $t \geqslant 1+x$ and, in particular, $\widehat{\zeta}(0, t)$ is uniformly bounded for $t \geqslant 1$. Similarly, using Lemma $6, \widehat{\alpha}(x, t)$ is also uniformly bounded for $t \geqslant 2(1-x)$ and, in particular, $\widehat{\alpha}(0, t)$ is uniformly bounded for $t \geqslant 2$.

Further, using the fact that

$$
\widetilde{\zeta}(x, t)=-\int_{x}^{1} \widetilde{\zeta}_{x}(\chi, t) d \chi+\widetilde{\zeta}(1, t)
$$

and from (44), it holds, for $x \in[0,1]$,

$$
\widetilde{\zeta}(x, t)^{2} \leqslant 5\left[\left\|\widetilde{\zeta}_{t}\right\|^{2}+\lambda^{2}\left(\|\widetilde{\zeta}\|^{2}+\|\widetilde{\omega}\|^{2}+\|\widehat{\zeta}\|^{2}+\|\widehat{\omega}\|^{2}\right)\right]
$$

Consequently, $\widetilde{\zeta}(x, t)$ is uniformly bounded for $t \geqslant 0$ and, in particular, $\widetilde{\zeta}(0, t)$ is uniformly bounded for $t \geqslant 0$. With similar arguments, as $\widetilde{\omega}(0, t)=-\widetilde{\zeta}(0, t)$ is uniformly bounded for $t \geqslant$ 0 , it holds that $\widetilde{\omega}(x, t)$ is uniformly bounded for $t \geqslant 0$, and, in particular, $\widetilde{\omega}(1, t)$ is uniformly bounded for $t \geqslant 0$. As $\mu(t)$ and $\widetilde{\omega}(1, t)$ are bounded for $t \geqslant 0$, one knows that $v(t)$ is bounded for $t \geqslant 1$. Therefore, from (46) and (76), $\widehat{\omega}(0, t)$ is uniformly bounded for $t \geqslant 1$, and, using Lemma $6, \widehat{\omega}(x, t)$ is uniformly bounded for $t \geqslant 1+x$.

Further, from (40)-(46), and (39),

$$
\begin{aligned}
\frac{d}{d t} v(t)^{2} & =2 a v(t)((q-1) v(t)+\widehat{\zeta}(0, t)+\widetilde{\zeta}(0, t) \\
& +v(t) \widetilde{q}(t)-\widetilde{d}(t)) \\
\frac{d}{d t}\|\widehat{\zeta}(t)\|^{2} & =\widehat{\zeta}(1, t)^{2}-\widehat{\zeta}(0, t)^{2}+2 \int_{0}^{1} \widehat{\zeta}(x, t) \hat{d}(t) d x \\
\frac{d}{d t}\|\widehat{\omega}(t)\|^{2} & =\widehat{\omega}(1, t)^{2}-\widehat{\omega}(0, t)^{2}+2 \int_{0}^{1} \widehat{\omega}(x, t) \hat{d}(t) d x \\
\frac{d}{d t}\|\widetilde{\zeta}(t)\|^{2} & =\widetilde{\zeta}(1, t)^{2}-\widetilde{\zeta}(0, t)^{2} \\
& +2 \lambda \int_{0}^{1} \widetilde{\zeta}(x, t)[\widetilde{\zeta}+\widetilde{\omega}+\widehat{\zeta}+\widehat{\omega}](x, t) d x \\
\frac{d}{d t}\|\widetilde{\omega}(t)\|^{2} & =\widetilde{\omega}(1, t)^{2}-\widetilde{\omega}(0, t)^{2} \\
& +2 \lambda \int_{0}^{1} \widetilde{\omega}(x, t)[\widetilde{\zeta}+\widetilde{\omega}+\widehat{\zeta}+\widehat{\omega}](x, t) d x \\
\frac{d}{d t} \widetilde{d}(t)^{2} & =-\widetilde{d}(t) \dot{\hat{d}}
\end{aligned}
$$

Using (9)-(10), Cauchy-Schwarz's inequality and the previous considerations, it is straightforward that the right-hand terms in the previous equations are all uniformly bounded for $t \geqslant 2$. Finally, integrating (96) from 0 to $\infty$, it follows that $\mu(t), \widetilde{d}_{\mu}(t),\|\widehat{\omega}(t)\|,\|\widehat{\zeta}(t)\|,\|\widetilde{\zeta}(t)\|,\|\widetilde{\omega}(t)\|,\left\|\widetilde{\zeta}_{t}\right\|$, and $\left\|\widetilde{\omega}_{t}\right\|$ are square integrable. Therefore, with $(183)$, so is $\widetilde{\omega}(1, t)$, and finally $v(t)$. Moreover, as $\widetilde{d}_{\mu}(t)$ and $\mu(t)$ are square integrable, so is $\widetilde{d}(t)$.

We conclude this proof with Barbalat's lemma. 


\section{REFERENCES}

[1] Z. Artstein, "Linear systems with delayed controls: a reduction," IEEE Transactions on Automatic Control, vol. 27, no. 4, pp. 869-879, 1982.

[2] M. Böhm, M. Krstic, S. Küchler, and O. Sawodny, "Modeling and boundary control of a hanging cable immersed in water," Journal of Dynamic Systems, Measurement, and Control, vol. 136(1), p. 011006 , 2013.

[3] D. Bresch-Pietri and M. Krstic, "Adaptive output feedback for oil drilling stick-slip instability modeled by wave PDE with anti-damped dynamic boundary," in IEEE American Control Conference, Portland, OR, June 2014, pp. 386-391.

[4] — "Adaptive output-feedback for wave PDE with anti-damping Application to surface-based control of oil drilling stick-slip instability," in IEEE Conference on Decision and Control, Los Angeles, CA, 2014, pp. 1295-1300

[5] - "Output-feedback adaptive control of a wave PDE with boundary anti-damping," Automatica, vol. 50, no. 5, pp. 1407-1415, 2014.

[6] F. Conrad and A. Mifdal, "Strong stability of a model of an overhead crane," Control and Cybernetics, vol. 27, no. 3, pp. 363-374, 1998.

[7] R. F. Curtain and H. Zwart, An introduction to infinite-dimensional linear systems theory. Springer Science \& Business Media, 2012, vol. 21.

[8] B. d'Andréa-Novel and J.-M. Coron, "Exponential stabilization of an overhead crane with flexible cable via a backstepping approach," Automatica, vol. 36, no. 4, pp. 587-593, 2000.

[9] N. Fourrier and I. Lasiecka, "Regularity and stability of a wave equation with a strong damping and dynamic boundary conditions," Evolution Equations \& Control Theory, vol. 2(4), pp. 631-667, 2013.

[10] P. A. Ioannou and J. Sun, Robust Adaptive Control. Prentice Hall, 1996.

[11] J. Jansen, "Nonlinear dynamics of oilwell drillstrings," Ph.D. dissertation, Delft University of Technology, 1993.

[12] J. Jansen and L. van der Steen, "Active damping of self-excited torsional vibrations in oil well drillstrings," Journal of Sound and Vibration, pp. 179(4):647-688, 1995.

[13] M. Krstic, "Adaptive control of an anti-stable wave PDE," in IEEE American Control Conference, Saint-Louis, MO, 2009, pp. 1505-1510.

[14] W. Kwon and A. Pearson, "Feedback stabilization of linear systems with delayed control," IEEE Transactions on Automatic Control, vol. 25, no. 2, pp. 266-269, 1980.

[15] A. Manitius and A. W. Olbrot, "Finite spectrum assignment problem for systems with delays," IEEE Transactions on Automatic Control, vol. 24, no. 4, pp. 541-552, 1979.

[16] T. Meurer and A. Kugi, "Tracking control design for a wave equation with dynamic boundary conditions modeling a piezoelectric stack actuator," International Journal of Robust and Nonlinear Control, vol. 21, pp. 542 - 562, 032011.

[17] S. Nicaise and C. Pignotti, "Exponential stability of second-order evolution equations with structural damping and dynamic boundary delay feedback," IMA Journal of Mathematical Control and Information, vol. 28, no. 4, pp. 417-446, 2011.

[18] G. Peralta and G. Propst, "Local well-posedness of a class of hyperbolic pde-ode systems on a bounded interval," Journal of Hyperbolic Differential Equations, vol. 11, no. 04, pp. 705-747, 2014.

[19] T. Ritto, C. Soize, and R. Sampaio, "Non-linear dynamics of a drillstring with uncertain model of the bit-rock interaction," International Journal of Non-Linear Mechanics, pp. 44(8):865-876, 2009.

[20] C. Roman, D. Bresch-Pietri, E. Cerpa, C. Prieur, and O. Sename, "Backstepping observer based-control for an anti-damped boundary wave PDE in presence of in-domain viscous damping," in IEEE 55th Conference on Decision and Control, Las Vegas, NV, Dec 2016, pp. 549-554.

[21] C. Roman, D. Bresch-Pietri, C. Prieur, and O. Sename, "Robustness of an adaptive output feedback for an anti-damped boundary wave PDE in presence of in-domain viscous damping," in IEEE American Control Conference, Boston, MA, July 2016, pp. 3455-3460.

[22] C. Sagert, F. Di Meglio, M. Krstic, and P. Rouchon, "Backstepping and flatness approaches for stabilization of the stick-slip phenomenon for drilling," in IFAC Systems Structure and Control, Grenoble, France, 2013, pp. 779-784.

[23] B. Saldivar, S. Mondié, and J. C. Ávila Vilchis, "The control of drilling vibrations: A coupled PDE-ODE modeling approach," International Journal of Applied Mathematics and Computer Science, vol. 26, no. 2, pp. 335-349, 2016.

[24] M. B. Saldivar, S. Mondie, J. J. Loiseau, and V. Rasvan, "Stick-Slip Oscillations in Oilwell Drillstrings: Distributed Parameter and Neutral
Type Retarded Model Approaches," in IFAC World Congress, Milano, Italy, 2011, pp. 18:284-289.

[25] A. Smyshlyaev, E. Cerpa, and M. Krstic, "Boundary stabilization of a 1-D wave equation with in-domain antidamping," SIAM Journal on Control and Optimization, vol. 48, no. 6, pp. 4014-4031, 2010.

[26] A. Smyshlyaev and M. Krstic, "Boundary control of an anti-stable wave equation with anti-damping on the uncontrolled boundary," Systems \& Control Letters, vol. 58, no. 8, pp. 617-623, 2009.

[27] Q.-Z. Zhang, Y.-Y. He, L. Li et al., "Sliding mode control of rotatory drilling system with stick slip oscillations," in Workshop on Intelligent Systems and Applications, Wuhan, China, 2010, pp. 1-4.

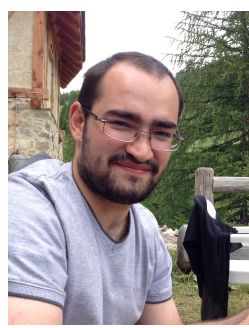

Christophe Roman was born in Val-des-près, France, in 1991. He graduated in applied physics and electrical engineering from the Ecole Normale Supérieur de Cachan, France in 2015. He received the $\mathrm{Ph}$. D degree in Automatic from the University Grenoble Alpes in 2018. He is currently working as a Post-doc at Gipsa-lab, Grenoble. His main research interest are the boundary feedback stabilization of partial differential equation, adaptive control, and model mismatch robustness.

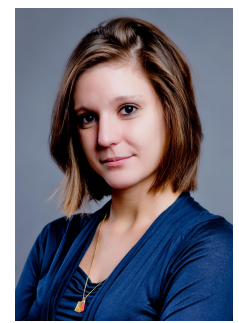

Delphine Bresch-Pietri received the M.S. degree in science and executive engineering and the Ph.D. degree in Mathematics and control from MINES ParisTech, Paris, France, in 2009 and 2012, respectively. From 2009 to 2012, she worked jointly between the Centre Automatique et Systms at MINES ParisTech and IFP Energies nouvelles. She is currently an Assistant Professor at the Systems and Control Center at MINES ParisTech. She is the coauthor of several patents in the field of engine control. Her research interests include engine control, theory and applications of time-delay systems, boundary control of partial differential equations and adaptive design.

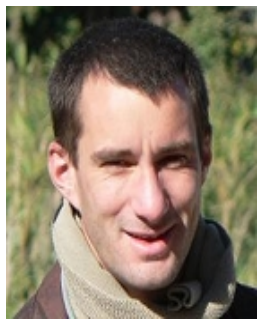

Christophe Prieur was born in Essey-les-Nancy, France, in 1974. He graduated in Mathematics from the Ecole Normale Supérieure de Cachan, France in 2000. He received the Ph.D degree in 2001 in Applied Mathematics from the Université ParisSud, France, and the "Habilitation Diriger des Recherches" (HDR degree) in 2009. From 2002 he was an associate researcher CNRS at the laboratory SATIE, Cachan, France, and at the LAAS, Toulouse, France (2004-2010). In 2010 he joined the Gipsalab, Grenoble, France where he is currently a senior researcher of the CNRS (since 2011). His current research interests include nonlinear control theory, hybrid systems, and control of partial differential equations.

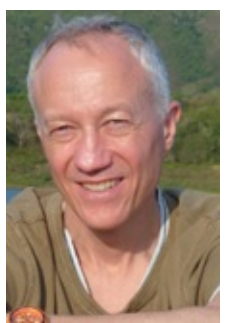

Olivier Sename received a $\mathrm{Ph} . \mathrm{D}$. degree from Ecole Centrale Nantes in 1994. He is now Professor at the Grenoble Institute of Technology within GIPSA-lab. His main research interests include Linear Parameter Varying systems and automotive applications. He is the (co-)author of 1 book, 48 international journal papers, and more than 200 international conference papers. He has led several industrial (Delphi, Renault, Volvo Trucks, JTEKT) and international (Mexico, Italy, Hungary) collaboration projects. He has supervised 27 Ph.D. students. 